\title{
Macroscopic fluctuations emerge in balanced networks with incomplete recurrent alignment
}

\author{
Itamar D. Landau (10) \\ Center for Brain Sciences, Hebrew University of Jerusalem, 9190401 Jerusalem, Israel \\ Haim Sompolinsky \\ Center for Brain Sciences, Hebrew University of Jerusalem, 9190401 Jerusalem, Israel; \\ Racah Institute of Physics, Hebrew University of Jerusalem, Jerusalem, Israel; \\ and Center for Brain Sciences, Harvard University, Cambridge, 02138 Massachusetts, USA
}

(Received 1 March 2021; accepted 10 May 2021; published 3 June 2021)

\begin{abstract}
Networks of strongly coupled neurons with random connectivity exhibit chaotic, asynchronous fluctuations. In a previous study, we showed that when endowed with an additional component of low-rank connectivity consisting of the outer product of orthogonal vectors, these networks generate macroscopic coherent fluctuations. Although a striking phenomenon, that result depended on a fine-tuned choice of low-rank structure. Here we extend that result by generalizing the theory of excitation-inhibition balance to networks with arbitrary low-rank structure and show that macroscopic fluctuations emerge intrinsically through what we call "incomplete recurrent alignment." We say that a low-rank connectivity structure exhibits incomplete alignment if its row space is not contained in its column space. In the generic setting of incomplete alignment, recurrent connectivity can be decomposed into a "subspace-recurrent" component and an "effective-feedforward" component. We show that the balance equations of excitation-inhibition networks generalize naturally to the setting of arbitrary strongly coupled low-rank structure and are determined by the subspace-recurrent component of connectivity. The effective-feedforward component, meanwhile, projects high-dimensional, microscopic fluctuations to a lowdimensional subspace where they are dynamically balanced by emergent macroscopic fluctuations. We present biologically plausible examples from excitation-inhibition networks and networks with heterogeneous degree distributions. Finally, we define the "alignment matrix" as the matrix of overlaps between left and right singular vectors of the structured connectivity, and show that the singular values of the alignment matrix determine the amplitude of macroscopic, low-dimensional variability, while its singular vectors determine the structure. Our work shows how low-dimensional fluctuations can emerge generically in strongly coupled networks with low-rank structure. Furthermore, by generalizing excitation-inhibition balance to arbitrary low-rank structure, our work may find relevance in any setting with strongly interacting units, whether in biological, social, or technological networks.
\end{abstract}

DOI: 10.1103/PhysRevResearch.3.023171

\section{INTRODUCTION}

The dynamic balance of excitation $(E)$ and inhibition $(I)$ is a paradigmatic theory for describing the activity of neocortical networks [1-4]. The theory describes how recurrent interactions generate asynchronous irregular firing activity similar to that typically observed in the cortex across many species, especially in awake, active states of behavior [5-9]. The $E-I$ balance network model is driven by strong feedforward excitation, and strong recurrent connectivity, dominated by inhibition, is necessary in order to balance that input. The result is that the average activity of $E$ and $I$ populations dynamically balance the mean synaptic input, enabling fluc-

\footnotetext{
*Present address: Department of Applied Physics, Stanford University, Stanford, CA 94305; itamar.landau@ mail.huji.ac.il

Published by the American Physical Society under the terms of the Creative Commons Attribution 4.0 International license. Further distribution of this work must maintain attribution to the author(s) and the published article's title, journal citation, and DOI.
}

tuations to propagate asynchronously. The resulting networks can account for various empirical observations of cortical activity, including not only irregular firing but also low pairwise correlations [4] and broad firing-rate distributions [10]. Furthermore, balanced networks perform fast tracking of external input which can underly predictive coding [11], and they are capable of amplifying input feature selectivity $[12,13]$ and generating stable patterns of activity for associative memory [14-16].

Many qualitative aspects of the dynamics of excitationinhibition balance can be understood by studying simplified firing-rate models [17]. Firing rate models of randomly connected excitatory and inhibitory populations with strong interactions and strong feedforward input exhibit a dynamic cancellation of the mean input at the population level $[18,19]$, similar to spiking models. The resulting state is chaotic and asynchronous due to the strong, asymmetric random connections [20].

It had been a longstanding question whether such randomly connected networks could intrinsically generate large-scale macroscopic fluctuations. We previously showed that when 
randomly connected networks are endowed with additional low-rank connectivity of a particular structure, macroscopic fluctuations emerge that are shared coherently across the entire network [21]. Specifically, connectivity structure consisting of outer products of orthogonal pairs embed a purely feedforward structure into the recurrent network such that high-dimensional, microscopic fluctuations along the row space are propagated to the column space, yielding shared, macroscopic variability in the column space, without generating feedback that would either suppress fluctuations or drive saturation. The same qualitative phenomenon was studied also by Darshan et al. [22] and Hayakawa and Fukai [23].

In the current work, we study a broader setting, which we refer to as "incomplete recurrent alignment," of which an orthogonal outer product is one limiting case. In order to develop our theory of incomplete recurrent alignment, we generalize the theory of excitation-inhibition balance to networks with arbitrary low-rank structure. A number of recent studies have explored the dynamics of networks with low-rank structured connectivity in addition to a random component of connectivity, yet these have all focused on weakly coupled low-rank structure $[24,25]$. We show that networks with strong low-rank connectivity structure operate in a dynamically balanced regime that is precisely analogous to $E-I$ networks: Without fine-tuning, population activity balances to cancel input currents and prevent saturation. This cancellation takes place in a specific linear subspace which we call the "balance subspace." As in E-I balance networks, activity orthogonal to the balance subspace exhibits microscopic chaotic fluctuations due to the random component of connectivity.

We show that macroscopic, coherent fluctuations in the balance subspace can be amplified by orders of magnitude depending on the structure of the low-rank connectivity, in particular the alignment between its left and right singular vectors. We say that a low-rank connectivity structure has incomplete alignment if the span of its right singular vectors (i.e., the row space) is not fully contained in the span of its left singular vectors (i.e., the column space). We show that the larger the extent of misalignment, the larger the fluctuations, and we derive a a second-order fluctuation-balance equation for these intrinsically generated macroscopic fluctuations. The amplitude and structure of macroscopic fluctuations is determined by the "alignment matrix," i.e., the matrix of overlaps between left and right singular vectors of the structured connectivity, while the time course of fluctuations is inherited from the time course of microscopic fluctuations.

In Sec. II, we introduce the model. In Sec. III, we present a decomposition of network activity into macroscopic order parameters that reside in the low-dimensional balance subspace on the one hand and microscopic degrees of freedom in the orthogonal subspace on the other. We show that strong lowrank connectivity yields dynamic balance-a linear equation for the macroscopic firing rates in the balance subspace and microscopic chaotic fluctuations in the orthogonal subspace. In Sec. IV, we show that incomplete alignment of the lowrank connectivity projects the microscopic fluctuations into the balance subspace, yielding amplified shared variability. We derive expressions for the amplitude, spatial structure, and timescale of this variability. Finally, in Sec. V, we study two concrete examples of biologically relevant incomplete alignment: excitatory-inhibitory networks with degenerate synaptic weight parameters (as previously studied in Ref. [26]) and networks with heterogeneous out-degrees.

\section{MODEL}

We study a network of $N$ firing-rate neurons with a connectivity matrix consisting of structured and random components. The structured component, $\mathbf{M}$, is given by a rank- $D$ matrix, where $D$ is finite in the large- $N$ limit, and the random component, J, has independent and identically distributed (i.i.d.) components assumed for simplicity to be Gaussian. Individual elements of both components of the connectivity scale as $\frac{1}{\sqrt{N}}$. Explicitly, we write the structured connectivity matrix in reduced singular value decomposition (SVD) form:

$$
\mathbf{M}=\frac{1}{\sqrt{N}} \mathbf{U} \mathbf{\Sigma} \mathbf{V}^{T}
$$

where both $\mathbf{U}$ and $\mathbf{V}$ are $N$-by- $D$ matrices with $O(1)$ components and orthogonal columns of norm $\sqrt{N}$, i.e., $\mathbf{U}^{T} \mathbf{U}=$ $\mathbf{V}^{T} \mathbf{V}=N \mathbf{I}_{D \times D}$ (notice that we adopt a scaling of $\mathbf{U}$ and $\mathbf{V}$ that differs from the standard SVD convention). $\boldsymbol{\Sigma}$ is a diagonal $D$-by- $D$ matrix with $O(1)$ positive elements, $\sigma_{k}$ with $k \in\{0,1 \ldots D-1\}$. Throughout this paper, boldface letters refer to vectors while boldface capital letters refer to matrices.

We define the random component, $\mathbf{J}$, to have elements sampled independently from $J_{i j} \sim \mathcal{N}\left(0, \frac{g^{2}}{N}\right)$ with a "gain" parameter $g=O(1)$. For simplicity, we assume that the elements of $\mathbf{J}$ are drawn independently of the structure of $\mathbf{U}$ and $\mathbf{V}$; i.e., we assume that $\frac{1}{N} \mathbf{V}^{T} \mathbf{J}^{k} \mathbf{U} \approx 0$ for all $k \geqslant 1$ [27].

The dynamical variables, $h_{i}$, are analogous to a neuron's membrane potential, and they are driven by strong external drive, $\sqrt{N} f_{i}$, with $f_{i} \sim O(1)$. Their dynamics are given by

$$
\frac{d \mathbf{h}}{d t}=-\mathbf{h}+(\mathbf{M}+\mathbf{J}) \mathbf{r}+\sqrt{N} \mathbf{f},
$$

where the firing rates of individual neurons are given instantaneously by $r_{i}=\phi\left(h_{i}\right)$, a sigmoidal nonlinearity. Simulations and numerical calculations in this paper use $\phi(h)=\tanh (h)$, which can be thought of as the change in firing rate relative to some baseline.

The input to the network from the structured component of connectivity can be written as $\mathbf{M r}=\frac{1}{\sqrt{N}} \sum_{k=0}^{D-1} \sigma_{k} \mathbf{u}_{k} \mathbf{v}_{k}^{T} \mathbf{r}$, where $\mathbf{u}_{k}$ and $\mathbf{v}_{k}$ are the columns of $\mathbf{U}$ and $\mathbf{V}$, respectively (i.e., the left and right singular vectors). This is displayed schematically in Fig. 1(a): Each right singular vector, $\mathbf{v}_{k}$, performs a readout of network activity that is fed back into the network along the corresponding left singular vector, $\mathbf{u}_{k}$, and scaled by $\sigma_{k}$.

\section{BALANCE SUBSPACE DECOMPOSITION AND THE ALIGNMENT MATRIX}

The span of the left-singular vectors, $\left\{\mathbf{u}_{k}\right\}_{k=0}^{D-1}$, i.e., the column-space of the structured connectivity $\mathbf{M}$, defines a distinct subspace. Both the structured and random components of connectivity have strong synapses, i.e., single elements are $O\left(\frac{1}{\sqrt{N}}\right)$. However, we observe that the SVD of the random 
(a)
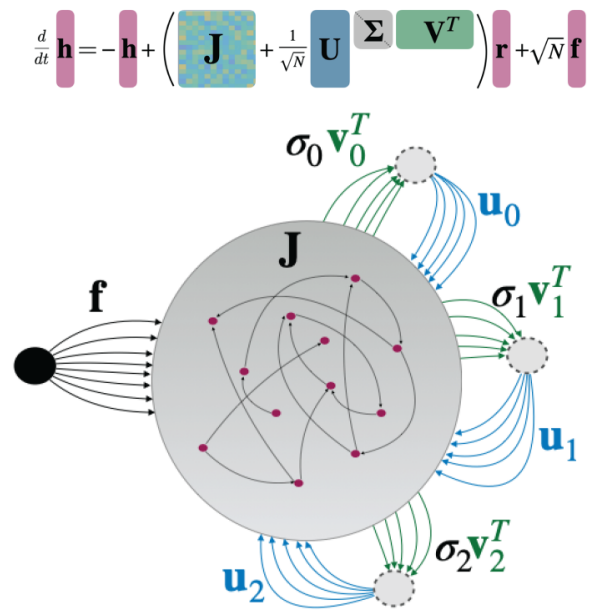

(c)

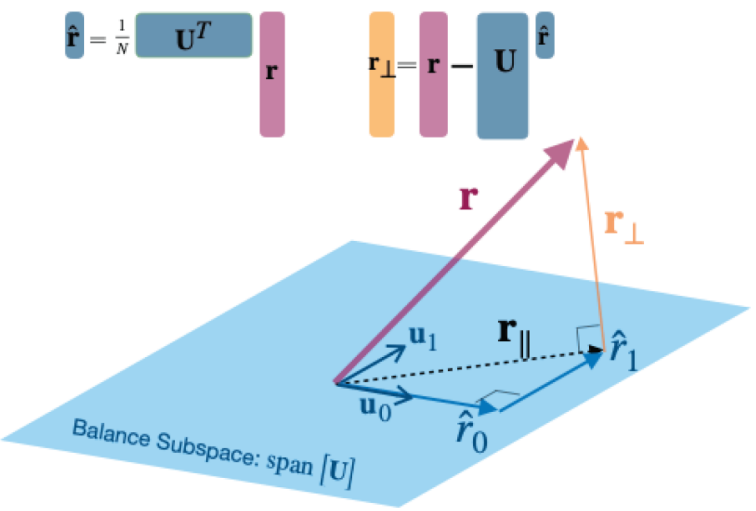

(b)

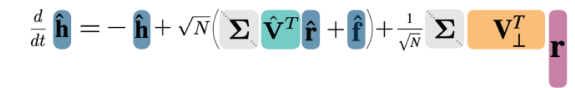

(d)

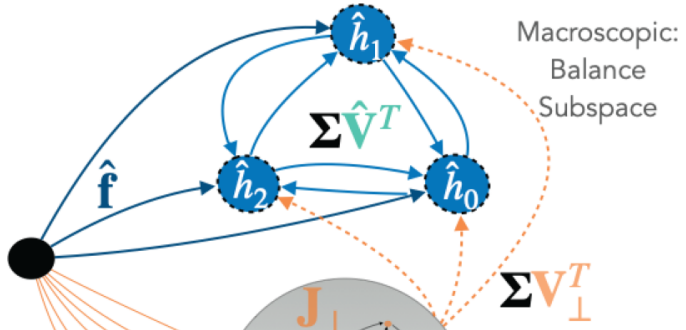

Microscopic:

Orthogonal

Complement

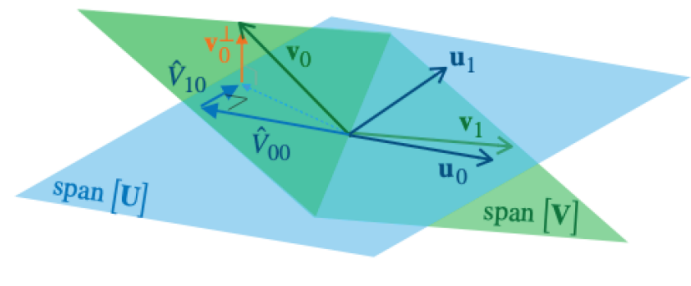

FIG. 1. Model dynamics and schematic. (a) Top: Graphical representation of the equations governing network dynamics [Eq. (2)]. Dynamical variables, $\mathbf{h}$, as well as firing rates, $\mathbf{r}$, and external drive, $\mathbf{f}$, are column vectors. Recurrent connectivity matrices include a random component, $\mathbf{J}$, and a strong low-rank component, $\mathbf{M}=\frac{1}{\sqrt{N}} \mathbf{U} \boldsymbol{\Sigma} \mathbf{V}^{T}$. Bottom: Schematic representation of network structure. Low-rank connectivity is represented as a read-out along each right-singular vector, $\mathbf{v}_{k}$, scaled by $\sigma_{k}$, and fed back to the network along left-singular vector, $\mathbf{u}_{k}$. (b) Balance subspace decomposition. Top: macroscopic dynamics given by the D-dimensional projection onto the balance subspace via $\hat{\mathbf{h}} \equiv \frac{1}{N} \mathbf{U}^{T} \mathbf{h}$. Bottom: Schematic decomposition. The subspace recurrence within the balance subspace is given by $\boldsymbol{\Sigma} \mathbf{V}^{T}$. The effectivefeedforward connectivity from the orthogonal complement to the balance subspace is given by $\boldsymbol{\Sigma} \mathbf{V}_{\perp}^{T}$. (c) Balance subspace decomposition for population vector of firing rates, $\mathbf{r}$. Top: Graphical representation of the definitions of the activity in the balance subspace $\hat{\mathbf{r}}$, and in the orthogonal complement $\mathbf{r}_{\perp}$. Bottom: Geometric visualization of the balance subspace decomposition. The projection into the balance subspace, $\mathbf{r}_{\|}$has components, $\hat{r}_{k}$, along each corresponding left-singular vector, $\mathbf{u}_{k}$. The same definitions apply for the dynamical variables, $\mathbf{h}$, and the external drive, f. (d) Balance subspace decomposition of the connectivity structure, defining the alignment matrix, $\hat{\mathbf{V}}$. Top: Equations defining $\hat{\mathbf{V}}$ and $\mathbf{V}_{\perp}$. Bottom: Geometric visualization of incomplete alignment. The span of the columns of $\mathbf{V}$ may generally not be constrained to the balance subspace. We display the projection of the first column, $\mathbf{v}_{0}$, onto the balance subspace and its components along each left-singular vector, $\mathbf{u}_{0}$ and $\mathbf{u}_{1}$, define the matrix elements $\hat{V}_{00}$ and $\hat{V}_{10}$, respectively. The projection of $\mathbf{v}_{0}$ onto the orthogonal complement defines, $\mathbf{v}_{0}^{\perp}$, the first column of $\mathbf{V}_{\perp}$. Similarly for $\mathbf{v}_{1}$, not shown. If the connectivity structure is "fully aligned", then the columns of $\mathbf{V}$ are obtained from the columns of $\mathbf{U}$ by rotation and reflection, such that the alignment matrix, $\hat{\mathbf{V}}$, is orthonormal.

component will yield singular values of $O\left(\frac{1}{N}\right)$, such that any vector of $O(1)$ firing rates will yield only $O(1)$ input via the random component. On the other hand, if $\mathbf{r}$ is aligned with $\mathbf{v}_{k}$, the structured component of connectivity will yield $O(\sqrt{N})$ input along $\mathbf{u}_{k}$. Therefore, the subspace spanned by the columns of $\mathbf{U}$ can receive strong recurrent inputs that will drive saturation unless they are balanced, and the components of the dynamics within this subspace will define the macroscopic order parameters of our system.

We call the column space of the structured connectivity the "balance subspace." We decompose the network activity and the connectivity into the balance subspace and its orthogonal complement. This is shown schematically in Fig. 1(b), and defined algebraically as follows: For any $N$-dimensional vector (or $N$-by- $D$ matrix), $\mathbf{X}$, we write $\mathbf{X}=\mathbf{X}_{\|}+\mathbf{X}_{\perp}$, where $\mathbf{X}_{\|}=\frac{1}{N} \mathbf{U} \mathbf{U}^{T} \mathbf{X}$ is the projection on the balance subspace, and $\mathbf{X}_{\perp}=\left(\mathbf{I}-\frac{1}{N} \mathbf{U} \mathbf{U}^{T}\right) \mathbf{X}$ is the projection in the orthogonal subspace. Within the balance subspace, we focus specifically on the components relative to $\mathbf{U}$, that is, we write $\mathbf{X}_{\|}=\mathbf{U} \hat{\mathbf{X}}$ with

$$
\hat{\mathbf{X}} \equiv \frac{1}{N} \mathbf{U}^{T} \mathbf{X} .
$$

The final decomposition is $\mathbf{X}=\mathbf{U} \hat{\mathbf{X}}+\mathbf{X}_{\perp}$. As an example, Fig. 1(c) shows both a graphical-algebraic representation and a geometric representation of $\hat{\mathbf{r}}$ and $\mathbf{r}_{\perp}$. We similarly decompose the input currents, $\mathbf{h}$, into $\mathbf{U h}+\mathbf{h}_{\perp}$.

Additionally, as will be motivated in the following section, we will decompose the matrix of right singular vectors of 
the structured connectivity, $\mathbf{V}=\mathbf{U} \hat{\mathbf{V}}+\mathbf{V}_{\perp}$, introducing the "alignment matrix," $\hat{\mathbf{V}}$, between the column space and row space of the structured connectivity,

$$
\hat{\mathbf{V}}=\frac{1}{N} \mathbf{U}^{T} \mathbf{V} .
$$

The alignment matrix is the $D$-by- $D$ matrix consisting of the overlaps between the right singular vectors and the left singular vectors. Figure 1(d) displays both a graphical-algebraic representation and a geometric representation of $\hat{\mathbf{V}}$, showing the geometry of two partially overlapping hyperplanes spanned by left and right singular vectors, respectively.

Importantly, if the right singular vectors are all contained in the balance subspace, i.e., if the row space is entirely contained in the column space, then the orthogonal complement is null $\left(\mathbf{V}_{\perp}=\mathbf{0}\right)$. We refer to this condition as "full recurrent alignment." Full recurrent alignment is obtained if and only if $\hat{\mathbf{V}}^{T} \hat{\mathbf{V}}=\mathbf{I}_{D \times D}$, i.e., if the alignment matrix is orthonormal.

We will assume the external drive is aligned with the balance subspace so that $\mathbf{f}=\mathbf{U} \hat{\mathbf{f}}$.

\section{A. Macroscopic dynamics in the balance subspace}

We decompose the dynamics [Eq. (2)], and first study the the coordinates of the dynamics within the balance subspace. These coordinates are the macroscopic order parameters of the network. To do so, we first apply the above decomposition [Eq. (3)] on the structured connectivity, $\mathbf{M}$ [Eq. (1)], in order to write $\hat{\mathbf{M}}=\frac{1}{\sqrt{N}} \boldsymbol{\Sigma} \mathbf{V}^{T}$. We note that by construction, $\mathbf{M}_{\perp}=$ 0 , since $\mathbf{M}$ projects entirely into the balance subspace.

In order to further decompose $\hat{\mathbf{M}}$, we use the alignment matrix to write $\mathbf{V}=\mathbf{U} \hat{\mathbf{V}}+\mathbf{V}_{\perp}$. This yields an important decomposition of the structured component of connectivity:

$$
\hat{\mathbf{M}}=\frac{1}{\sqrt{N}} \boldsymbol{\Sigma} \hat{\mathbf{V}}^{T} \mathbf{U}^{T}+\frac{1}{\sqrt{N}} \boldsymbol{\Sigma} \mathbf{V}_{\perp}^{T} .
$$

The first term comprises the "subspace-recurrent" component of the structured connectivity, which is entirely recurrent to the balance subspace. The second is the "effectivefeedforward" component, which propagates activity from the orthogonal subspace to the balance subspace.

Given population firing rates, $\mathbf{r}=\mathbf{U} \hat{\mathbf{r}}+\mathbf{r}_{\perp}$, the subspacerecurrent input is $\sqrt{N} \boldsymbol{\Sigma} \hat{\mathbf{V}}^{T} \hat{\mathbf{r}}$ and the effective-feedforward input is $\frac{1}{\sqrt{N}} \boldsymbol{\Sigma} \mathbf{V}_{\perp}^{T} \mathbf{r}_{\perp}$. Projecting the dynamics [Eq. (2)] onto the balance subspace yields the dynamics of the macroscopic order parameters:

$$
\frac{d \hat{\mathbf{h}}}{d t}=-\hat{\mathbf{h}}+\sqrt{N}\left(\boldsymbol{\Sigma} \hat{\mathbf{V}}^{T} \hat{\mathbf{r}}+\hat{\mathbf{f}}\right)+\frac{1}{\sqrt{N}} \boldsymbol{\Sigma} \mathbf{V}_{\perp}^{T} \mathbf{r}_{\perp}+O\left(\frac{1}{\sqrt{N}}\right),
$$

where the $O\left(\frac{1}{\sqrt{N}}\right)$ term is a contribution from the random connectivity, $\mathbf{J}$, onto the balance subspace, which we have ignored. Note that the effective-feedforward contribution via $\mathbf{V}_{\perp}$ is of $O(1)$.

In order to avoid saturation, the balance subspace activity, $\hat{\mathbf{r}}$, must reach a fixed point that cancels the large, $O(\sqrt{N})$, input term to leading order [as displayed graphically in Fig. 2(a)]. This balanced fixed point is governed by $D$ (a)

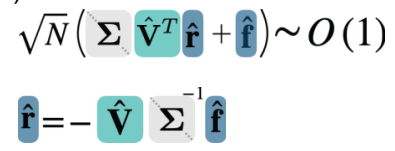

(b)
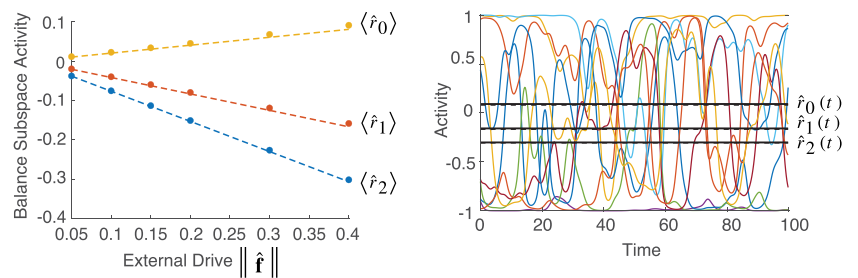

FIG. 2. Dynamic balance in networks with low-rank structure. (a) Top: Graphical algebraic representation of the $D$-dimensional balance conditions on the activity in the balance subspace in order to cancel potentially large input, and below, the resulting solution to the linear balance equations in the case of full alignment of the structured connectivity (i.e., when the alignment matrix, $\hat{\mathbf{V}} \equiv \frac{1}{N} \mathbf{U}^{T} \mathbf{V}$, is orthonormal). Bottom: Balance subspace time-average activity, $\left\langle\hat{r}_{k}\right\rangle$, vs strength of external drive, given by the norm of its projection onto the balance subspace, $\|\hat{\mathbf{f}}\|$. Solid circles mark simulation results, which match closely with the prediction from linear balance equations, shown in dotted lines. Multiple instantiations of Gaussian $\mathbf{U}$ result in near identical $\left\langle\hat{r}_{k}\right\rangle$ (10 instantiations here are indistinguishable). (b) Top: Dynamical equations for the microscopic degrees of freedom in the orthogonal subspace. Bottom: Sample activity trace. Colored lines are individual neuron firing rates from a random sample of 10 neurons, $r_{i}(t)$, displaying chaotic fluctuations. Black lines are the balance subspace firing rates, $\hat{r}_{k}(t)$, with simulations in solid lines and theory in dashed lines.

(approximately) linear equations:

$$
\boldsymbol{\Sigma} \hat{\mathbf{V}}^{T} \hat{\mathbf{r}}^{*}+\hat{\mathbf{f}}=O\left(\frac{1}{\sqrt{N}}\right) .
$$

Note that the effective-feedforward input does not contribute to the balance fixed point up to leading order. It will nevertheless have an impact on the macroscopic fluctuations around the fixed point, as we shall see below.

The linear balance equations derived here generalize the two-dimensional $E$-I balance equations [2] to arbitrary lowrank structure. In our formalism, the balance equations have a natural basis in the columns of $\mathbf{U}$, i.e., the left singular vectors of the structured component of connectivity. Moreover, these balance equations are independent of the structure of $\mathbf{U}$, depending only on the singular values, $\boldsymbol{\Sigma}$, and the alignment matrix, $\hat{\mathbf{V}}$.

Figure 2(a) displays the solution to the balance equations for the case in which $\hat{\mathbf{V}}$ is orthonormal, and the result of simulations confirming that the balance subspace firing rates are well predicted by the linear balance equations, independently of $\mathbf{U}$.

Finally, we note that in general the balance equations may or or may not have an obtainable solution (e.g., consistent with saturating local rates). In the following, we will assume that the external inputs are chosen such that the balance equations yield a feasible solution; see Appendix A. 


\section{B. Microscopic dynamics in the orthogonal subspace}

We now consider the dynamics in the subspace orthogonal to the balance subspace by projecting the full dynamics [Eq. (2)] via $\mathbf{h}_{\perp}=\left(\mathbf{I}-\frac{1}{N} \mathbf{U} \mathbf{U}^{T}\right) \mathbf{h}$ (recall that we assume that the external drive is contained in the balance subspace):

$$
\frac{d \mathbf{h}_{\perp}}{d t}=-\mathbf{h}_{\perp}+\mathbf{J}_{\perp} \mathbf{r} .
$$

These equations are displayed graphically in Fig. 2(b). Due to the random connectivity in the orthogonal subspace, $\mathbf{J}_{\perp}=$ $\left(\mathbf{I}-\frac{1}{N} \mathbf{U U}^{T}\right) \mathbf{J}$, these microscopic dynamics can be described by a Gaussian dynamic mean-field theory (DMFT) which we detail in the Appendix B (and which follows [19,20]). The mean-field theory admits a fixed point or an asynchronous, chaotic state. The theory predicts that as long as the balance equations are satisfied and saturation is avoided [Eq. (7)], sufficiently strong random connectivity will yield chaotic fluctuations.

The order parameter that captures the statistics of the microscopic dynamics is the mean single-neuron autocorrelation function

$$
C(\tau) \equiv\left[\left\langle\delta r_{i}(t) \delta r_{i}(t+\tau)\right\rangle\right],
$$

where \langle\rangle is time-average, $\delta r_{i}=r_{i}-\left\langle r_{i}\right\rangle$, and [] is average over neurons. The dynamic mean-field theory yields a differential equation governing $C(\tau)$, which is detailed in Appendix B 2.

Note that the microscopic dynamics and mean-field theory differential equation for $C(\tau)$ depend on the macroscopic dynamics of the balance subspace, $\hat{\mathbf{h}}$. Specifically, the state of the macroscopic dynamics determines each neuron's set point in the nonlinearity, via $\mathbf{r}=\phi\left(\mathbf{U} \hat{\mathbf{h}}+\mathbf{h}_{\perp}\right)$. The macroscopic dynamics, in turn, depend on the statistics of the microscopic dynamics, specifically on their total variance (see Appendix A). Thus, the mean-field theory gives a set of self-consistency equations that determine both the fixed point values of $\hat{\mathbf{h}}$ and the total variance of $\mathbf{h}_{\perp}$ that together will yield balance subspace firing rates that satisfy the balance equations, Eq. (7). These dynamic mean-field equations simplify in the case of Gaussian $\mathbf{U}$ (see Appendix C), and we solve them numerically and find a good match between simulation and theory (see Fig. 5 in Appendix C).

Figure 2(b) shows an example activity trace from this chaotic balance state, in which single unit activity fluctuates greatly while balance subspace activity stays constant, satisfying the balance equations.

As mentioned above, we assume that the balance firing rates given by Eq. (7) yield a nonsaturating solution, and further, that that the random component of connectivity is sufficiently strong so as to yield chaotic microscopic dynamics (see Appendix B).

\section{INCOMPLETE ALIGNMENT AMPLIFIES FLUCTUATIONS IN THE BALANCE SUBSPACE}

In this section, we assume a network in the chaotic balanced state described above. In this state, the macroscopic balance subspace firing rates, $\hat{\mathbf{r}}$ [defined via Eq. (3)], satisfy the balance equations [Eq. (7)]. As we shall shortly see, in the case of fully aligned structured connectivity, balance requires that $\hat{\mathbf{r}}$ are constant to leading and subleading order, i.e., with fluctuations $O\left(\frac{1}{N}\right)$. In this section, we show that incomplete alignment amplifies macroscopic fluctuations substantially: Any non-negligible misalignment will yield macroscopic fluctuations that are larger by a factor of $O(\sqrt{N})$.

We study the fluctuations of the activity in the balance subspace, relative to its time average (indicated by angle brackets):

$$
\delta \hat{\mathbf{r}}(t) \equiv \hat{\mathbf{r}}(t)-\langle\hat{\mathbf{r}}\rangle .
$$

The statistics of these fluctuations are quantified by the $D$-by$D$ matrix of covariance functions:

$$
\hat{\mathbf{C}}(\tau) \equiv\left\langle\delta \hat{\mathbf{r}}(t) \delta \hat{\mathbf{r}}^{T}(t+\tau)\right\rangle .
$$

We first consider the case of full alignment, in which $\mathbf{V}_{\perp}=$ $\mathbf{V}-\mathbf{U} \hat{\mathbf{V}}=0$, and there is no effective feedforward input from the orthogonal subspace to the balance subspace. By subtracting the time average from Eq. (6), we see that the dynamics of $\delta \hat{\mathbf{h}} \equiv \hat{\mathbf{h}}-\langle\hat{\mathbf{h}}\rangle$ are then given by

$$
\frac{d \delta \hat{\mathbf{h}}}{d t}=-\delta \hat{\mathbf{h}}+\sqrt{N} \boldsymbol{\Sigma} \hat{\mathbf{V}}^{T} \delta \hat{\mathbf{r}}+O\left(\frac{1}{\sqrt{N}}\right),
$$

where the $O\left(\frac{1}{\sqrt{N}}\right)$ contribution is from the random connectivity, and we remind the reader that the alignment matrix, $\hat{\mathbf{V}}=\frac{1}{N} \mathbf{U}^{T} \mathbf{V}$, is orthonormal in the case of full alignment.

In this case, we must have $\sqrt{N} \delta \hat{\mathbf{r}} \ll 1$; otherwise the input fluctuations driving $\delta \hat{\mathbf{h}}$ will be $O(1)$, and this will destabilize the balance state. We verify this numerically and find that in fully aligned networks the total variance of temporal fluctuations, given by the trace of $\hat{\mathbf{C}}(0)$, is $O\left(\frac{1}{N^{2}}\right)$ (as also discussed recently [11]).

To probe the impact of misalignment, we simulate networks with varying levels of alignment (see Appendix F 1 for details) and varying network size. We show in Fig. 3(a) that in misaligned networks the macroscopic fluctuations are amplified significantly: Their total variance is $O\left(\frac{1}{N}\right)$. Furthermore, as displayed in Fig. 3(b), we find that incomplete alignment yields nontrivial covariance structure, whereas in fully aligned networks $\hat{\mathbf{C}}$ is essentially structureless.

To explain the emergence of macroscopic fluctuations, we return to the dynamics of $\delta \hat{\mathbf{h}}$, in the general, $\mathbf{V}_{\perp} \neq 0$, case:

$$
\frac{d \delta \hat{\mathbf{h}}}{d t}=-\delta \hat{\mathbf{h}}+\boldsymbol{\Sigma}\left(\sqrt{N} \hat{\mathbf{V}}^{T} \delta \hat{\mathbf{r}}+\hat{\boldsymbol{\eta}}\right),
$$

where $\hat{\boldsymbol{\eta}}=\frac{1}{\sqrt{N}} \mathbf{V}_{\perp}^{T} \delta \mathbf{r}_{\perp}$ are the fluctuations in the effective feedforward input from the orthogonal subspace to the balance subspace due to the misaligned connectivity. If the typical elements of $\mathbf{V}_{\perp}$ are $O(1)$, then microscopic fluctuations in the orthogonal subspace are projected to the balance subspace yielding input fluctuations, $\hat{\eta}$, that are also $O(1)$. Thus, we see that incomplete alignment will drive significant macroscopic input correlations. Indeed, as we show for an example network in Fig. 3(c), the input fluctuations to the balance subspace from the orthogonal subspace are $O(1)$.

A stable balanced state requires that these $O(1)$ effective feed-forward input fluctuations from $\hat{\boldsymbol{\eta}}$ be canceled to leading order by recurrent balance subspace fluctuations in $\delta \hat{\mathbf{r}}$. If these are not canceled, but instead propagate to drive $O(1)$ fluctuations in $\delta \hat{\mathbf{h}}$, then the balance equations will be violated. Thus, 
(a)

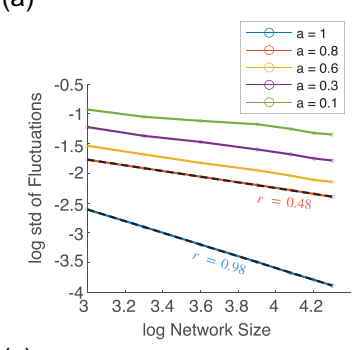

(c)
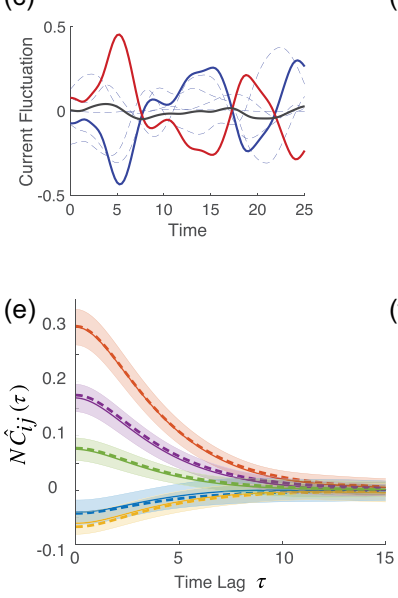

(b)

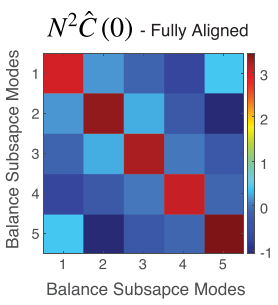

(d)

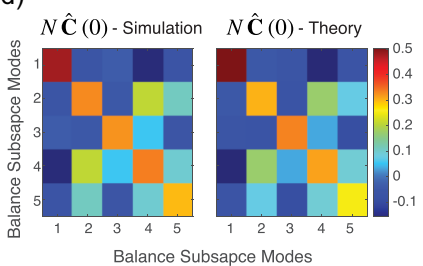

(f)

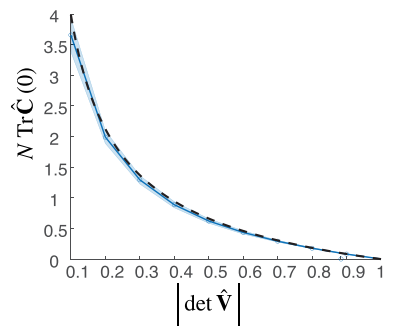

FIG. 3. Incomplete misalignment increases macroscopic fluctuations in the balance subspace. (a) Log-log plot of total macroscopic temporal fluctuations vs network size, i.e., $\frac{1}{2} \log _{10} \operatorname{Tr} \hat{\mathbf{C}}(0)$ vs $\log _{10} N$, for varying values of alignment parameter, $a$ ( $a=1$ is fully aligned; see Appendix F 1 for details). Linear fit (dashed line) shows that standard deviation of fluctuations scales as $\frac{1}{\sqrt{N}}$ for networks with incomplete alignment, as compared to $\frac{1}{N}$ for fully aligned networks $(D=3)$. (b) The $D$-by- $D$ covariance of firing rate fluctuations in the balance subspace, $\hat{\mathbf{C}}(0)$, for a fully aligned network is structureless. Compare panel (d) for case of incomplete alignment. (c) Sample trace of fluctuations in input currents onto a single mode of the balance subspace $(D=5, N=8000) . O(1)$ fluctuations in the "effective feedforward" input from the orthogonal subspace (red) are canceled by fluctuations in the "subspace-recurrent input" from within the balance subspace (blue, dotted lines each display input from individual modes and solid line displays net subspace-recurrent input fluctuations). Block solid line displays net fluctuations in input current, which are $O\left(\frac{1}{\sqrt{N}}\right)$. (d) $D$-by- $D$ covariance of firing rate fluctuations in the balance subspace, displayed here scaled by $N$ [i.e., $N \hat{\mathbf{C}}(0)]$. Left: single trial simulation. Right: Theory given by Eq. (14). (e) Crosscorrelation functions from all $D=5$ "presynaptic" modes, scaled by $N, N \hat{C}_{i j}(\tau)$. Red is the autocorrelation; other colors display cross correlation with other modes. Dotted lines show theory [Eq. (14)]. Solid lines show simulation results with shaded regions displaying standard deviation over 20 random realizations of $\mathbf{U}$ for fixed $\hat{\mathbf{V}}$. In panels (c) and (d), $|\operatorname{det} \hat{\mathbf{V}}|=0.2255$, and the singular values of $\hat{\mathbf{V}}$ were set to be $\{0.9,0.825,0.75,0.675,0.6\}$ (see Appendix F2 for details). (f) Total variance of firing rate fluctuations in the balance subspace, scaled by $N$, plotted against the determinant of the alignment matrix, $\hat{\mathbf{V}}$. Blue shows simulation results with standard deviation over 20 random realizations. Dashed black line shows theory as given by Eq. (15). In these simulations, the external drive, f, was rescaled in order to fix the norm of the balance firing rates, $\left\|\hat{r}^{*}\right\|$ (see Appendix F 2 and Fig. 5 for more details), $g=2.5, D=5$, and $N=10000$ in all simulations unless otherwise noted.

in order to maintain $O\left(\frac{1}{\sqrt{N}}\right)$ fluctuations in $\delta \hat{\mathbf{h}}$, the balance subspace activity must satisfy the following set of fluctuation balance equations:

$$
\sqrt{N} \hat{\mathbf{V}}^{T} \delta \hat{\mathbf{r}}+\hat{\boldsymbol{\eta}}=O\left(\frac{1}{\sqrt{N}}\right),
$$

requiring $\delta \hat{\mathbf{r}} \approx-\frac{1}{\sqrt{N}}\left(\hat{\mathbf{V}}^{T}\right)^{-1} \hat{\boldsymbol{\eta}}$ to leading order.

The fluctuation balance equation [Eq. (13)] allows us to derive an analytical expression for the amplitude, structure, and temporal profile of the fluctuations in $\delta \hat{\mathbf{r}}$. As we show in Appendix D,

$$
\hat{\mathbf{C}}(\tau)=\frac{C(\tau)}{N}\left(\left[\hat{\mathbf{V}} \hat{\mathbf{V}}^{T}\right]^{-1}-\mathbf{I}\right),
$$

where $C\left[(\tau) \equiv\left\langle\delta r_{i}(t) \delta r_{i}(t+\tau)\right\rangle\right]$ is the average singleneuron autocorrelation [Eq. (9)], which can be calculated by dynamic mean-field theory given the balance fixed point, $\hat{\mathbf{r}}^{*}$ (see Appendix B 2 and Fig. 5).

The total temporal variance in the balance subspace is

$$
\left\langle\delta \hat{\mathbf{r}}^{T} \delta \hat{\mathbf{r}}\right\rangle=\frac{C(0)}{N} \sum_{k=1}^{D}\left(\frac{1}{s_{k}^{2}}-1\right),
$$

where $s_{k}$ are the singular values of the alignment matrix, $\hat{\mathbf{V}}$, showing that decreased alignment, as measured by decreased singular values of the alignment matrix, increases the macroscopic temporal fluctuations. Furthermore, we can see that as a network approaches full alignment, $s_{k} \rightarrow 1$, the $O\left(\frac{1}{N}\right)$ leading contribution to the net macroscopic fluctuations vanishes, consistent with our observation [Fig. 3(a)] of $O\left(\frac{1}{N^{2}}\right)$ scaling in fully aligned networks.

Our analytical expression [Eq. (14)] reveals how misalignment, via $\hat{\mathbf{V}}$, imprints a nontrivial spatial structure on the fluctuations in the balance subspace. From Eq. (14) (see also Appendix D), one sees that the eigenvectors of the cross covariance, $\hat{\mathbf{C}}$, i.e., the principal components of the balance subspace activity, are given by the left singular vectors of the alignment matrix, $\hat{\mathbf{V}}$. The corresponding eigenvalues are determined by the corresponding singular values of $\hat{\mathbf{V}}$, and given by $\frac{C(0)}{N}\left(\frac{1}{s_{k}^{2}}-1\right)$. Thus, the spatial structure of macroscopic fluctuations is entirely determined by the singular value decomposition of the alignment matrix. Interestingly, it is independent of $\boldsymbol{\Sigma}$, the singular values of the full structured connectivity, because $\Sigma$ multiplies [Eq. (12)] both the effective-feedforward input, $\hat{\eta}$, and the balance subspace recurrent input, $\hat{\mathbf{V}}^{T} \delta \hat{\mathbf{r}}$, and therefore does not enter the fluctuation balance equation [Eq. (13)].

Another notable feature of Eq. (14) is that $\hat{\mathbf{C}}(\tau)$ is a product of a $D$-by- $D$ matrix and a scalar temporal profile, and thus the time course of macroscopic fluctuations are identical across the $D$ modes of the balance subspace and are given by the average single-neuron autocorrelation function, $C(\tau)$.

In order to verify our predictions, we construct networks with Gaussian $\mathbf{U}$ and a heterogeneous set of $\left\{s_{k}\right\} \in[0,1]$ [Figs. 3(d)-3(f); see Appendix F 2 for details]. For our choice of parametrization, we derive an expression for the total temporal variance as a function of $\operatorname{det} \hat{\mathbf{V}}=\prod_{k} s_{k}$ and verify it over an order of magnitude for different instantiations of $\hat{\mathbf{V}}$ with randomly chosen singular vectors [Fig. 3(f)]. In 
Figs. 3(d)-3(e), we show that for a given choice of $\hat{\mathbf{V}}$, both our closed-form expression for the spatial structure [Fig. 3(d)] and our numerically computed, theoretical prediction of the time course [Fig. 3(e)] yield very good predictions.

For a fixed network size, the macroscopic fluctuations grow with decreased alignment until at least one singular value, $s_{j}$, is on the order of magnitude of $\frac{1}{\sqrt{N}}$. As we detail in Appendix E, at that scale, the activity of the corresponding mode is unconstrained by the leading-order balance equations [Eq. (7)]. At the same time, the recurrent input generated by fluctuations in that same mode are not sufficient to fully cancel the effective feedforward fluctuations, $\hat{\eta}$, and the fluctuation balance equation [Eq. (13)] cannot be satisfied. In that situation, the theory derived here breaks down, and macroscopic synchronous fluctuations with $\delta \hat{\mathbf{h}}, \delta \hat{\mathbf{r}} \sim O(1)$ can emerge. Similar scenarios were studied in Refs. [22,23]).

A full self-consistent theory describing the fluctuations in $\hat{\mathbf{h}}$ is beyond the scope of this work.

\section{BIOLOGICALLY RELEVANT EXAMPLES}

\section{A. Degenerate excitation-inhibition balance}

We first provide a sketch of the application of our generalized balance framework in the well-known setting of excitation-inhibition balance. Typically such a network is constructed by randomly and independently assigning connections to a fraction, $p$, of all pairs of neurons, with the synaptic weight (and sign) from neuron $j$ to neuron $i$ depending on each of their identities as either excitatory or inhibitory:

$$
W_{i j}=\left\{\begin{array}{ll} 
\pm \frac{J_{\alpha_{i} \alpha_{j}}}{p \sqrt{N}} & \text { with prob } p \\
0 & \text { with prob } 1-p
\end{array},\right.
$$

where $\alpha_{i}=I$ for $N_{I}$ inhibitory neurons and $\alpha_{i}=E$ for the remaining $N_{E}$ excitatory neurons, and the sign of the weight is corresponding to the presynaptic neuron, so that if $\alpha_{j}=E(I)$ the $j$ th column of $W$ is positive (negative). Such a random binary connectivity matrix can be approximated by a lowrank, deterministic component with a 2-by-2 block structure and an additional random component [19]. Despite not being symmetric (or normal), the low-rank component is in general fully aligned: The balance subspace is the two-dimensional subspace spanned by two block vectors, one with matching signs and one with opposing signs:

$$
\begin{aligned}
& \mathbf{u}_{0} \sim\left(\begin{array}{l}
+ \\
+
\end{array}\right), \\
& \mathbf{u}_{1} \sim\left(\begin{array}{c}
+ \\
-
\end{array}\right) .
\end{aligned}
$$

That is, the balance subspace consists of a "sum mode" and a "difference mode" of the excitatory and inhibitory populations. The readout performed by the structured connectivity is from the same subspace; that is, the span of $\left\{\mathbf{v}_{0}, \mathbf{v}_{1}\right\}$ is identical and therefore the alignment matrix is orthonormal.

However, consider the situation in which the synaptic weight is independent of the identity of the postsynaptic neuron: $J_{E I}=J_{I I}=J_{I}$ and $J_{E E}=J_{I E}=J_{E}$. Because the average synaptic strengths in this network do not depend on the identity of the postsynaptic neuron, the structured component of connectivity is rank 1: It is the outer product of a sum mode and a difference mode. As Helias et al. [26] have also shown, this parametrization yields amplified fluctuations in both the $\mathrm{E}$ and I populations. We now show that this these fluctuations can be characterized as a specific case of our theory of incomplete alignment.

For simplicity, we assume $N_{I}=N_{E}=\frac{N}{2}$ (see Appendix $\mathrm{G} 1$ for general case). Explicitly, we write the structured component of connectivity as $\frac{\sigma}{\sqrt{N}} \mathbf{u}_{0} \mathbf{v}_{0}^{T}$, according to our generalized balance formalism, with the following definitions:

$$
\begin{aligned}
\mathbf{u}_{0} & =\left(\begin{array}{l}
\mathbf{1} \\
\mathbf{1}
\end{array}\right), \\
\mathbf{v}_{0} & =\frac{1}{\sigma}\left(\begin{array}{c}
J_{E} \mathbf{1} \\
-J_{I} \mathbf{1}
\end{array}\right), \\
\sigma & =\sqrt{\frac{J_{E}^{2}+J_{I}^{2}}{2},}
\end{aligned}
$$

where $\mathbf{1}$ is the uniform column vector of length $\frac{N}{2}$.

We observe that the alignment matrix in this case is just a scalar given by the overlap between $\mathbf{u}_{0}$ and $\mathbf{v}_{0}$ :

$$
\hat{v}=\frac{1}{N} \mathbf{u}_{0}^{T} \mathbf{v}_{0}=\frac{J_{E}-J_{I}}{2 \sigma} .
$$

Inhibition dominance and network stability require that $J_{I} \geqslant$ $J_{E}$. We assume the external drive is uniform, $\mathbf{f}=\mathbf{1} r_{0}$, and then the balance equation yields population average firing

$$
\hat{r}=-\frac{r_{0}}{\hat{v} \sigma}=\frac{2 r_{0}}{J_{I}-J_{E}} .
$$

To avoid saturation while changing the $I / E$ ratio, the external drive must shrink to compensate for diminished alignment, by scaling $r_{0} \propto \hat{v} \sigma=J_{I}-J_{E}$.

The macroscopic order parameter of the balance subspace is the "sum mode," i.e., simply the average network activity over both $E$ and $I$. The right singular vector, $\mathbf{v}_{0}^{T}$, however, reads out from both the sum mode and also from the difference mode. The difference between $E$ and $I$ activity, which is unconstrained by balance, fluctuates randomly and will be tightly balanced by fluctuations in population firing rate.

Applying our theory [Eq. (14)], fluctuation balance yields the following expression for the autocorrelation of the population firing rate, $\hat{C}(\tau)=\langle\hat{r}(t) \hat{r}(t+\tau)\rangle$ :

$$
\hat{C}(\tau)=\frac{C(\tau)}{N}\left(\frac{J_{E}+J_{I}}{J_{E}-J_{I}}\right)^{2} .
$$

As the relative strength of inhibition decreases toward parity with excitation, the alignment between $\mathbf{v}_{0}$ and $\mathbf{u}_{0}$ shrinks: The right singular vector, $\mathbf{v}_{0}^{T}$, reads out less from the sum mode, and therefore larger population fluctuations are necessary to balance the growing contribution from fluctuations of the difference mode. By fixing $J_{E}$ and varying the ratio $\frac{J_{I}}{J_{E}}$, while scaling $r_{0}$ to maintain a fixed balance firing rate, we confirm this prediction via simulations in Fig. 4(a).

In the limit of $J_{I} \rightarrow J_{E}$, the expression for $\hat{C}(\tau)$ diverges. In that limit, the size of the fluctuations along $\mathbf{u}_{0}$, i.e., coherent fluctuations shared by the entire population, is $O(1)$, as detailed in Appendix E. Fluctuation balance breaks down as $\hat{r}$ no longer drives sufficient subspace-recurrent input. 

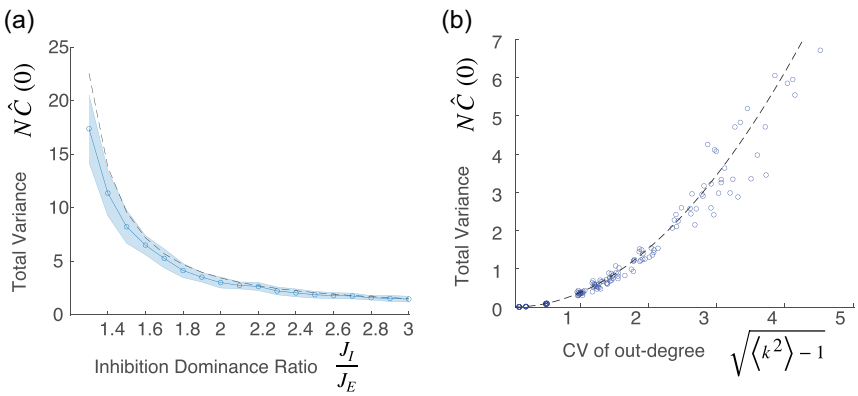

FIG. 4. Biologically relevant examples of incomplete alignment yielding low-dimensional fluctuations: (A) $E-I$ network with degenerate weight parameters in the sense that the strength of both $E$ and $I$ synapses are independent of the identity of the postsynaptic neuron. Variance of population firing rate over time, scaled by $N=20000$ in this simulation. Dotted black line shows theory [Eq. (24)]. Shaded region shows standard error of the mean over 20 trials. (b) Inhibitory network with heterogeneous out degree. Variance of the population firing rate over time, scaled by $N$, as a function of the coefficient of variation $(\mathrm{CV})$ of the the out degree. Dotted line shows theory [Eq. (31)]. Circles show single trial simulations. Out degrees were drawn from a log-normal distribution. In degrees were taken to be uniform. $N=10000$.

Furthermore, once the fluctuations in $\hat{r}$ are of $O(1)$, they begin to significantly impact the fluctuations in the orthogonal complement, making them non-Gaussian and impacting their timescale, as studied in Refs. [21,23].

\section{B. Heterogeneous out degrees}

We now employ our theory of misalignment to study the dynamics of networks with heterogeneous out degrees (see Appendix G 2 for the case of both heterogeneous out and in degrees). Consider a single inhibitory population in which each neuron $i$ has $K_{i}$ randomly chosen outgoing connections, and average degree $K=p N$ for some $O(1)$ value of $p$. We suppose each nonzero synapse has weight $-\frac{J}{\sqrt{N}}$. Just as in the $E / I$ setting, such a random binary connectivity structure can be approximated by a deterministic low-rank component and a random component of connectivity. The deterministic component in this case can be written as

$$
M_{i j}=-\frac{J}{\sqrt{N}} \frac{K_{j}}{N} .
$$

This deterministic $\mathbf{M}$ is a rank-1 matrix given by $\mathbf{M}=\sigma \mathbf{u}_{0} \mathbf{v}_{0}^{T}$. Defining $k_{i}$ as the vector of relative out degrees, $k_{i} \equiv \frac{K_{i}}{K}$, and $\left[k^{2}\right]$ as the mean square of the relative out degrees, as well as $p$ as the mean probability of connection $p \equiv \frac{K}{N}$, we have the following definitions:

$$
\begin{gathered}
\mathbf{u}_{0}=\mathbf{1}, \\
\mathbf{v}_{0}=-\frac{\mathbf{k}}{\sqrt{\left[k^{2}\right]}}, \\
\sigma_{0}=\sqrt{\left[k^{2}\right]} J p,
\end{gathered}
$$

where $\mathbf{1}$ is the column vector of all ones.
The alignment matrix is scalar in this case as well and is given by

$$
\hat{v}=-\frac{\mathbf{1}^{T} \mathbf{k}}{N} \frac{1}{\sqrt{\left[k^{2}\right]}}=-\frac{1}{\sqrt{\left[k^{2}\right]}} .
$$

Thus, we see that the extent of alignment and therefore the strength of subspace recurrence decrease with increasing breadth of the out-degree distributions.

As in the previous example, we assume the external drive is uniform, $\mathbf{f}=\mathbf{1} r_{0}$, and the balance equation gives

$$
\hat{r}=-\frac{r_{0}}{\hat{v} \sigma}=\frac{r_{0}}{J p}
$$

such that the balance firing rates in the population are unaffected by broadening of the out degree. This is intuitive because the mean recurrent input is not expected to depend on the breadth of the out degree.

Nevertheless, broad out-degree distribution increases the extent of macroscopic fluctuations. The right singular vector $\mathbf{v}_{0}$ reads out from both the population average sum mode and also from a mode that is weighted by out degree with high-degree neurons contributing positively and lowdegree neurons negatively. Broader out degrees correspond to a smaller contribution of the sum mode, requiring larger population fluctuations to balance the larger drive from the degree-weighted mode. Concretely, we have

$$
\hat{C}(\tau)=\frac{C(\tau)}{N}\left(\left[k^{2}\right]-1\right) .
$$

Note that $\left[k^{2}\right]-1$ is the variance of the relative out degrees. The increase of correlated fluctuations with broader out degree was observed in Ref. [28].

We find that broader out-degree distribution leads to larger coherent fluctuations and confirm this prediction via simulations [Fig. 4(b)]. It is worth noting that the structure of $\mathbf{V}_{\perp}=\mathbf{V}-\mathbf{U} \hat{V}$ does not enter the mean-field theory, beyond the assumption that $\boldsymbol{\eta}=\frac{1}{\sqrt{N}} \mathbf{V}_{\perp}^{T} \delta \mathbf{r}$ is an independent meanzero Gaussian process. In practice, we generate out degrees from a log-normal distribution and find that the simulations fit the theory well within a broad range of out-degree variability, although the fit worsens as the variability increases, as presumably the Gaussian assumption is violated.

In Appendix G 2, we show that in networks with heterogeneous in degrees as well, introducing negative correlations between in and out degrees will further amplify the shared fluctuations.

\section{DISCUSSION}

We have generalized the theory of dynamic excitationinhibition balance to networks with arbitrary low-rank structured connectivity, $\mathbf{M}=\frac{1}{\sqrt{N}} \mathbf{U} \mathbf{\Sigma} \mathbf{V}^{T}$, together with an additive random component of connectivity. We decompose the network dynamics into the "balance subspace," given by $\operatorname{span}(\mathbf{U})$, which receives strong structured recurrent input, and its orthogonal complement within which the recurrent input is driven by random connectivity. The core insight of our theory is that the macroscopic, low-dimensional dynamics in the balance subspace are determined by the alignment matrix, $\hat{\mathbf{V}}=$ 
$\frac{1}{N} \mathbf{U}^{T} \mathbf{V}$, between left and right singular vectors. We derive linear balance equations for the activity in the balance subspace which are a generalization of the two balance equations in excitation-inhibition networks. These balance equations are independent of the underlying statistics of the balance subspace itself, $\mathbf{U}$, but rather depend only on the alignment, $\hat{\mathbf{V}}$, and the singular values, $\boldsymbol{\Sigma}$, that scale the recurrent connectivity in each of the balance subspace modes.

We observe that $\hat{\mathbf{V}}$ quantifies the alignment between row space and column space of the structured connectivity, that is, between the the readout and feedback subspaces. If the readout subspace is identical to the feedback subspace, then $\hat{\mathbf{V}}$ is orthonormal. If $\hat{\mathbf{V}}$ is not an orthonormal matrix, then the structured connectivity is not fully aligned. In this case, the structured connectivity can be decomposed to a subspacerecurrent component that reads out network activity strictly from within the balance subspace and an effective feedforward component that reads out network activity from the orthogonal subspace and projects it to the balance subspace.

We show that increased misalignment increases coherent, low-dimensional fluctuations in the balance subspace. This is due to the strengthening of the effective feedforward component of the structured connectivity, which reads out microscopic fluctuations from the orthogonal complement and projects them to the balance subspace. It is important to emphasize that the fluctuating input currents projected from the orthogonal complement to the balance subspace are of $O(1)$ but that balance leads to a dynamic canceling of these fluctuating currents to leading order. This cancellation is manifested by a set of fluctuation-balance equations that enable us to determine the size, structure, and temporal profile of firing rate fluctuations in the balance subspace, $\hat{\mathbf{C}}(\tau)$, as a function of $\hat{\mathbf{V}}$. This cancellation of fluctuations is analogous to the cancellation of feed-forward cancellation studied previously $[4,26]$, except that here the input fluctuations are intrinsically generated through the chaotic dynamics in the orthogonal subspace and projected onto the balance subspace via the misaligned structured connectivity. Note that those studies, as well as Ref. [22], presented the cancellation as a set of balance equations on the correlation structure of the activity whereas we have instead presented our theory via fluctuationbalance equations. These two perspectives on second-order balance are equivalent.

Despite the suppression of fluctuations via dynamic balance, the magnitude of coherent fluctuations due to misalignment is $O(\sqrt{N})$ larger than under fully aligned connectivity. These fluctuations increase with misalignment as a function of the singular values of $\hat{\mathbf{V}}$, because as the subspace-recurrent feedback is weakened larger macroscopic fluctuations in the balance subspace are needed in order to cancel the input fluctuations from the orthogonal complement. Because they are driven by the microscopic fluctuations in the orthogonal complement, the temporal profile of these shared macroscopic fluctuations is identical to the average temporal profile of single-neuron fluctuations. The spatial structure of the fluctuations within the $D$-dimensional balance subspace is given by the left-singular vectors of the alignment matrix.

Note that in the limit where at least one of the singular values of $\hat{\mathbf{V}}$ is zero, fluctuation balance cannot be achieved along the corresponding modes. Any external drive into these modes will drive saturation, but if there is no external drive to these modes, then the fluctuations in the orthogonal complement will be propagated to this mode via the structured connectivity in a fully feed-forward manner, without being suppressed by dynamic balance. This can lead to $O(1)$ shared fluctuations, as studied in Ref. [21].

We have studied continuous rate-neuron models for their analytical tractability. However, we expect that our theory will hold qualitatively for spiking-neuron network models as well. This is because we find that despite the elevated shared fluctuations, networks with incomplete alignment exhibit asynchronous dynamics as long as the singular values of $\hat{\mathbf{V}}$ are larger than $O\left(\frac{1}{\sqrt{N}}\right)$. Thus, we expect that spiking networks with strong low-rank connectivity structure will operate in an asynchronous, irregular regime that is well described by a mean-field theory [3], which can be extended to include the impact of misaligned structure.

We have studied the structure of temporal fluctuations but our approach can be readily extended to study the structure of quenched variability over multiple realizations of the random connectivity. Incomplete alignment will amplify quenched fluctuations in a similar manner.

Achieving a balanced fixed point requires that the external drive be almost entirely contained in the balance subspace [with only up to $O\left(\frac{1}{\sqrt{N}}\right)$ projections onto the orthogonal subspace], and imposes additional constraints on $\hat{\mathbf{f}}$ so that the balance equations yield a nonsaturating solution. For example, in standard $E-I$ balance networks, external drive onto the $E$ population must be sufficiently stronger than that onto the $I$ population in order to maintain non-negative firing rates [2,29]. Describing these constraints in the general lowrank setting is beyond the scope of this work.

Throughout this work, we assume that $\hat{\mathbf{f}}$ is chosen such that it enables a nonsaturating balanced fixed point and that the "gain," $g$, of the random component of connectivity is sufficient to drive chaotic fluctuations. We note that decreasing alignment, while maintaining a fixed $\hat{\mathbf{f}}$ and $g$, will effectively weaken the subspace recurrent input to the balance subspace. This will result in shifting the balance fixed point to larger magnitude firing rates and eventually suppress chaos and drive saturation, as we showed in Ref. [30].

We note that the random component of connectivity may have nontrivial statistical structure; for example, different cell types may have different overall variability of their synaptic strengths, yielding a block structure on the variances [19,27,31]. Additionally, the low-rank structure may be correlated with the random component of connectivity [27]. These types of correlations in the connectivity may have nontrivial impact in the regime of dynamic balance, which may be of interest for future study.

\section{A. Relationship to previous models of dynamic balance}

Our framework of generalized dynamic balance unifies much previous work. For example, the typical $E-I$ balance network has rank-2 deterministic structure, in which both the column space and row space consist of the same pair of modes (a "sum" mode and a "difference" mode), and therefore the structure is fully aligned. Some $E-I$ network models, 
however (e.g., Refs. [3,32]), use a degenerate block structure by constraining the average weights to be independent of postsynaptic population. As we have shown here, in this case, the structured connectivity has rank 1 and is only partially aligned. The balance subspace is a sum mode, $\mathbf{u}_{0} \propto \mathbf{1}$, while the readout mode, $\mathbf{v}_{0}$, is a combination of sum and difference modes. As the relative strength of inhibition decreases, $\mathbf{v}_{0}$ becomes dominated by the difference mode and thus the connectivity becomes less aligned and yields larger coherent fluctuations. These increased fluctuations in the case of degenerate $E$-I structure have been previously studied by Helias et al. [26]. As suggested in the discussion in Ref. [26], even stronger correlations arise in the case of a "doubly degenerate" $E-I$ structure, in which in addition to being independent of postsynaptic population the average weights are set to zero. Such a case was studied in Ref. [23], corresponds to the limit where $\hat{\mathbf{V}}=0$ in our formalism, and is equivalent to the setting studied in Ref. [21].

$E-I$ networks with distance dependent connectivity have been the focus of a number of past studies [14,22,33-35]. In the typically studied setting, each of the $E$ and $I$ connectivity profiles share the same periodic boundary conditions and are constrained to finite spatial frequency modes, and therefore both the column space and row space are spanned by a concatenated Fourier basis of $E$ and $I$ cells. Thus, the recurrent structure is fully aligned and $\hat{\mathbf{V}}$ is orthonormal. Huang et al. [34] studied shared variability that can arise via spatiotemporal pattern formation in such models when the balance state is destabilized. We study networks in a stable balance regime.

Darshan et al. [22] studied how shared variability can arise intrinsically in $E-I$ balanced networks with distancedependent connectivity. They showed that singular low-rank structure can yield significant shared variability. For example, they consider the case in which the $I$-to- $E$ connectivity has spatial dependence while all other connectivity profiles are spatially uniform. In our formalism, this means the column space (columns of $\mathbf{U}$ ) no longer includes Fourier modes of the $I$ population but rather consists of Fourier modes of the $E$ population concatenated with the zero vector over the $I$ population. On the other hand, the row space (columns of $\mathbf{V}$ ) does not include Fourier modes from the $E$ population, but rather consists of $I$-population Fourier modes concatenated with the zero vector over the $E$ population, and therefore these modes are entirely orthogonal to $\mathbf{U}$ so that they are fully feed-forward (at least of one the singular values of $\hat{\mathbf{V}}$ is zero). Thus spatially correlated fluctuations are propagated from the $I$ population to the $E$ population, where they are not canceled. As further discussed in Ref. [22] in Sec. IV C, the situation is different if the $I$ population has distant-dependent connectivity internally as well. In our formalism, this would mean that while columns of $\mathbf{V}$ would still not include Fourier modes of $E$, the columns of $\mathbf{U}$ would once again consist of the concatenated $E$ and $I$ Fourier modes. Thus, this is an example of partial alignment. Therefore, the spatially correlated fluctuations in the presence of $I$-to- $I$ distance dependence are an order of magnitude smaller than in the case where only $I$-to- $E$ is distance dependent. These fluctuations are still an order of magnitude larger than in the fully aligned case in which the $E$ population also has distance-dependent projections (whether to itself or to the $I$ population).

Networks with heterogeneous in degrees have been previously shown to exhibit broken balance [36,37]. That result can be understood in a straightforward manner in our framework of generalized balance: If the in degrees from the external drive are not a linear combination of $E$ and $I$ in degrees, then the external drive is not fully aligned with the balance subspace and there will be strong external drive to the orthogonal complement which will drive saturation [30]. Here we have studied the impact of heterogeneous out degrees on dynamic balance. We show that broad out-degree distributions in balanced networks are a form of incomplete alignment and result in increased coherent fluctuations. A similar phenomenon was observed numerically in Ref. [28]. We provide an analytical expression for the size of fluctuations as a function of the breadth of the out degrees and verify it numerically. We furthermore show that negative correlations between in and out degrees will further amplify the shared fluctuations (Appendix G 2).

Other work has shown that balance networks can exhibit shared variability if they have shared fluctuations in their external input $[38,39]$. In our framework, those studies amount to introducing external drive fluctuations that are orthogonal to the balance subspace, which therefore are not canceled. Our work assumes the external input is constrained to the balance subspace and constant in time.

\section{B. Conclusion}

Previous studies have explored particular examples of low-rank deterministic structure in balanced networks, most often via distance-dependent connectivity [14,22,33,39] or sub-population structure $[19,38]$. In such low-rank structures, the column space (the span of the left singular vectors) is typically identical to the row space (the span of the rightsingular vectors). We call such networks "fully aligned" and study the more general situation of partial alignment in which the row space may not be entirely contained in the column space of the low-rank matrix. We show that such incomplete alignment has qualitative impact on network dynamics. The key feature of the structured connectivity in our analysis is the alignment matrix, composed of the overlaps between left- and right-singular vectors.

Low-rank structured connectivity may reflect different cell types, distance dependence, functional connectivity, and heterogeneity between neurons $[14,22,33,37]$. Such a generalization may be important for incorporating biological realism into balance-network models. From another perspective, lowrank structure has been of recent interest in designing networks that perform specific computations [25,40]. Our work studies a new regime where low-rank connectivity is strong and suggests a bridge between networks designed for computation and biological networks exhibiting dynamic balance.

We have developed a generalized theory of dynamic balance, which both unifies previous studies and reveals new results. Our generalization expands the study of dynamic balance to a broad class of low-rank structures: those with 
only partial alignment between column space and row space. These structures have previously only appeared in particular cases, but in our framework they appear as the general case of low-rank connectivity. We show that incomplete alignment generates coherent fluctuations via effective feed-forward propagation from a high-dimensional subspace with microscopic chaos to a low-dimensional, balance subspace. We derive a set of fluctuation balance equations that provides an analytical solution for the structure of coherent fluctuations in the balance subspace.

This theory may find relevance well beyond neuroscience. Recent studies of complex systems attempt to explore the relationship between structure and dynamics in a variety of real-world networks [41,42]. Many of these studies limit the strength of interaction between units $\left(\sim \frac{1}{N}\right)$ in order to facilitate mean-field approaches. The theory of excitationinhibition balance studies a regime of strong interactions $\left(\sim \frac{1}{\sqrt{N}}\right)$ but until now its application has remained limited to neuroscience because of the excitation-inhibition structure (Dale's law). Our generalized framework of dynamic balance may be relevant for any setting with strongly interacting units, whether biological, social, or technological networks.

All simulations and numerical solutions were performed using MATLAB. Code is available at GitHub [43].

\section{ACKNOWLEDGMENTS}

We thank David Hansel and Yoram Burak for useful comments on previous versions of this manuscript. This research was partially supported by the Swartz Program in Theoretical Neuroscience at Harvard, the Gatsby Charitable Foundation, and NIH Grant NINDS (Grant No. 1U19NS104653).

\section{APPENDIX A: SELF-CONSISTENCY OF THE BALANCE SOLUTION}

As discussed in the main text, the macroscopic dynamics in the balanced subspace [Eq. (6)] admit a balanced fixed point governed by the $D$ linear balance equations: $\boldsymbol{\Sigma} \hat{\mathbf{V}}^{T} \hat{\mathbf{r}}^{*}+\hat{\mathbf{f}} \approx 0$ [Eq. (7) in the main text]. Balance is achieved when the firing rates in the balance subspace satisfy this equation up to a finite-size correction of $\frac{1}{\sqrt{N}}$. These firing rates, $\hat{\mathbf{r}}$, are given by the components of the full population firing rate vector, $\mathbf{r}=\phi(\mathbf{h})$, along $\mathbf{U}$ :

$$
\hat{\mathbf{r}}^{*}=\frac{1}{N} \mathbf{U}^{T} \phi\left(\mathbf{U} \hat{\mathbf{h}}^{*}+\mathbf{h}_{\perp}\right) .
$$

As we shall see, these $D$ equations constrain both the macroscopic dynamical variables in the balance subspace, $\hat{\mathbf{h}}^{*}$, as well as the statistics of the microscopic, local dynamics in the orthogonal complement, $\mathbf{h}_{\perp}$.

The dynamical state in the orthogonal complement can be either a stable fixed point (FP) or chaos. Given a fixed $\hat{\mathbf{h}}^{*}$, those dynamics are described by a mean-field theory that predicts that the microscopic degrees of freedom, $h_{\perp_{i}}$, can be described as independent, identical, mean-zero Gaussians, either fixed in time or fluctuating with a monotonically decaying autocorrelation. The mean-field theory follows previous work (e.g., Refs. $[19,20]$ ), and we detail it for our general low-rank setting below in Appendix B. The result of the mean-field theory is that if balance is achieved, avoiding saturation, then there is a FP with Gaussian statistics that transitions to chaos for sufficiently strong random component of connectivity. The expression for the variance in the orthogonal subspace, $\Delta_{0}=\left\langle h_{\perp_{i}}^{2}\right\rangle$, depends only on $\hat{\mathbf{h}}^{*}$ and $g$. In the FP regime, $\Delta_{0}$ is the spatial disorder and is given by a single implicit equation, while in the chaotic regime, $\Delta_{0}$ averages both spatial and temporal disorder, and together with the spatial variance of the single-neuron time averages, it is constrained by a pair of equations.

Given $\Delta_{0}, \hat{\mathbf{r}}$ is given by first averaging over the Gaussian component in the orthogonal subspace and then projecting the result onto balance subspace,

$$
\hat{\mathbf{r}}^{*}=\frac{1}{N} \mathbf{U}^{T}\left\langle\phi\left(\mathbf{U} \hat{\mathbf{h}}^{*}+\sqrt{\Delta_{0}} z\right)\right\rangle_{D z},
$$

where \langle\rangle$_{D z}$ is an integral over the standard normal measure, $D z=\frac{d z}{2 \pi} e^{-\frac{z^{2}}{2}}$. Combining the balance requirement of Eq. (7) gives us a set of $D$ implicit equations which, given $\Delta_{0}$, determine $\hat{\mathbf{h}}^{*}$ :

$$
\frac{1}{N} \mathbf{U}^{T}\left\langle\phi\left(\mathbf{U} \hat{\mathbf{h}}^{*}+\sqrt{\Delta_{0}} z\right)\right\rangle_{D z}=-\hat{\mathbf{V}} \boldsymbol{\Sigma}^{-1} \hat{\mathbf{f}} .
$$

We stress that this Gaussian mean-field equation for selfconsistency in the balance subspace holds regardless of whether the dynamics in the orthogonal subspace are in the FP or chaotic regime, but the total variance in the orthogonal subspace, $\Delta_{0}$, must also be found in a manner self-consistent with $\hat{\mathbf{h}}^{*}$, as we show below.

\section{APPENDIX B: MEAN-FIELD THEORY IN THE ORTHOGONAL SUBSPACE}

We now detail the mean-field theory describing the dynamics in the orthogonal subspace for both fixed point and chaos. We will assume an approximate fixed point in the balance subspace, $\hat{\mathbf{h}}^{*}$, that enables the corresponding firing rates, $\hat{\mathbf{r}}^{*}$, to satisfy the $D$ balance equations [Eq. (7)].

We further assume full external alignment, as we have throughout the main text, so that the external drive, $\mathbf{f}$, does not project into the orthogonal subspace. The dynamics are given by

$$
\frac{d \mathbf{h}_{\perp}}{d t}=-\mathbf{h}_{\perp}+\mathbf{J}_{\perp} \mathbf{r},
$$

where, we remind the reader, $\mathbf{X}_{\perp}=\left(\mathbf{I}-\frac{1}{N} \mathbf{U} \mathbf{U}^{T}\right) \mathbf{X}$ is the projection of the vector or matrix of column vectors, $\mathbf{X}$, onto the orthogonal subspace, and that the vector of firing rates is given by $\mathbf{r}=\phi(\mathbf{h})$. Through the nonlinearity, $\phi$, the dynamics in the orthogonal complement depend on the dynamics in the balance subspace, $\hat{\mathbf{h}}^{*}$, because $\mathbf{h}=\mathbf{U} \hat{\mathbf{h}}+\mathbf{h}_{\perp}$.

\section{Fixed point}

The fixed point equation in the orthogonal subspace is

$$
\mathbf{h}_{\perp}^{*}=\mathbf{J}_{\perp} \mathbf{r}^{*}
$$

Given a fixed $\hat{\mathbf{h}}^{*}$, we follow the mean-field theory presented, for example, in Ref. [19], which treats the recurrent drive due to the random connectivity, as independent, identical, mean-zero Gaussians. The matrix, $\mathbf{J}_{\perp}$, is the projection of 
the random matrix, $\mathbf{J}$, into the orthogonal subspace. Because elements of $\mathbf{J}$ are assumed to be drawn independently of the structure of $\mathbf{U}$, the projected random matrix, $\mathbf{J}_{\perp}$, has uncorrelated elements, and therefore we can assume $\left(\sum_{j}^{N} J_{\perp_{i j}} r_{j}\right)^{2} \approx$ $\sum_{j} J_{\perp_{i j}}^{2} r_{j}^{2} \approx N\left[J_{i j}^{2}\right]\left[r_{j}^{2}\right]$, just as in the original theory (where [ ] is average over neurons). Note that the variance of single elements of $\mathbf{J}_{\perp}$ is actually smaller than the variance of the full random matrix by a factor $1-\left(\frac{D}{N}\right)^{2}$, but we ignore this factor because $D$ is assumed $O(1)$ in the large- $N$ limit.

The mean-field therefore determines the spatial variance in the orthogonal subspace at the fixed point, $\Delta_{0} \equiv\left[h_{\perp_{i}}^{2}\right]$, to be

$$
\Delta_{0}=g^{2}\left[r_{j}^{2}\right] \text {. }
$$

The mean-square firing rates, $\left[r_{j}^{2}\right]=\left[\phi^{2}\left(h_{j}\right)\right]$, must be found by averaging over the network, where $h_{j}$ has a component in the balance subspace and an additional Gaussian component with variance $\Delta_{0}$. Because the two components are independent, we can perform the average over the Gaussian randomness before averaging over the population, and thus we arrive at an implicit mean-field fixed-point equation for $\Delta_{0}$ :

$$
\Delta_{0}=g^{2} \frac{\mathbf{1}^{T}}{N}\left\langle\phi^{2}\left(\mathbf{U} \hat{\mathbf{h}}^{*}+\sqrt{\Delta_{0}} z\right)\right\rangle_{D z},
$$

where \langle\rangle$_{D z}$ means averaging over the standard normal measure $D z=e^{-\frac{z^{2}}{2}} d z$.

\section{Dynamic mean-field theory for chaos in the orthogonal subspace}

In the chaotic regime, the input to the orthogonal subspace can still be considered Gaussian but its temporal statistics must be derived. That is, we seek to find the autocorrelation:

$$
\Delta(\tau) \equiv\left[\left\langle h_{i}^{\perp}(t) h_{i}^{\perp}(t+\tau)\right\rangle\right] .
$$

The dynamics in the orthogonal subspace can be represented by a single stochastic differential equation:

$$
\frac{d h_{i}^{\perp}}{d t}=-h_{i}^{\perp}+\eta_{i},
$$

where $\eta_{i}(t)=\sum_{j} J_{i j}^{\perp} r_{j}(t)$. Again, due to the randomness of the connectivity, $\eta_{i}$ has mean-zero over neurons and time, and the average autocorrelation of $\eta_{i}$ is a scaled version of the average autocorrelation of $r_{i}$ :

$$
\left[\left\langle\eta_{i}(t) \eta_{i}(t+\tau)\right\rangle\right]=g^{2} C_{T}(\tau),
$$

where we have written

$$
C_{T}(\tau) \equiv\left[\left\langle\phi\left(h_{i}(t)\right) \phi\left(h_{i}(t+\tau)\right)\right\rangle\right]
$$

for the average autocorrelation of the firing rates. Note that we have inserted the notation $C_{T}$ for the total autocorrelation in order to differentiate from the mean single-neuron temporal autocorrelation $C(\tau)=C_{T}(\tau)-\left[\left\langle\phi\left(h_{i}\right)\right\rangle^{2}\right]$ introduced in the main text in Sec. III B, Eq. (9), and used in Sec. IV, Eq. (14).

To compute $C_{T}(\tau)$, we first write $h_{i}=\mathbf{u}_{i}^{T} \hat{\mathbf{h}}^{*}+h_{i}^{\perp}$, where $\mathbf{u}_{i}$ is the $i$ th row of $\mathbf{U}$ and gives the projection of neuron $i$ in the balance subspace. Next, we rewrite the two correlated Gaussians, $h_{i}^{\perp}(t)$ and $h_{i}^{\perp}(t+\tau)$, via three independent Gaussians, one of which contributes the correlated component:

$$
h_{i}^{\perp}(t)=\sqrt{\Delta_{0}-\Delta(\tau)} x_{1}+\sqrt{\Delta(\tau)} y,
$$

$$
h_{i}^{\perp}(t+\tau)=\sqrt{\Delta_{0}-\Delta(\tau)} x_{2}+\sqrt{\Delta(\tau)} y,
$$

where we have introduced $\Delta_{0} \equiv \Delta(0)$ as the total variance. These three Gaussians need to be integrated over and then the balance subspace structure averaged to yield

$C_{T}(\tau)=\frac{1}{N} \sum_{i}\left\langle\left\langle\phi\left(\mathbf{u}_{i}^{T} \hat{\mathbf{h}}^{*}+\sqrt{\Delta_{0}-\Delta(\tau)} x+\sqrt{\Delta(\tau)} y\right)\right\rangle_{D x}^{2}\right\rangle_{D y}$.

Thus, given the autocorrelation, $\Delta(\tau)$, in the orthogonal complement, we have an expression for the average singleneuron firing rate autocorrelation, $C_{T}(\tau)$. Next, following the standard DMFT approach $([19,20])$, we write an implicit differential equation that determines the autocorrelation selfconsistently:

$$
\left(1-\frac{\partial^{2}}{\partial \tau^{2}}\right) \Delta(\tau)=g^{2} C_{T}(\tau)
$$

The total variance, $\Delta_{0}$, is the initial condition of Eq. (B12) and must be found self-consistently along with the conditions $\frac{\partial}{\partial \tau} \Delta(0)=0$ and $\frac{\partial^{2}}{\partial \tau^{2}} \Delta(\infty)=0$. As detailed in $[19,20]$, the differential equation [Eq. (B12)] can be re-expressed as a onedimensional dynamics under a potential energy. Given $\hat{\mathbf{h}}$, the initial condition, $\Delta_{0}$, can be found by using the requirement that the potential energy at the initial condition equals its value at $\tau \rightarrow \infty$, together with the fact that $\Delta(\infty)=g^{2} C_{T}(\infty)$. Therefore, in practice, given the fixed point in the balance subspace, $\hat{\mathbf{h}}^{*}$, the total variance in the orthogonal subspace, $\Delta_{0}=\left[\left\langle\left(h_{i}^{\perp}\right)^{2}\right\rangle\right]$, and the spatial variance of the time averages, $\Delta(\infty)=\left[\left\langle h_{i}^{\perp}\right\rangle^{2}\right]$, are found via a pair of coupled equations. The fixed point in the balance subspace depends on $\Delta_{0}$ in turn, via the balance equations [Eq. (A3)].

In sum, the mean-field characterization of the system determines $\hat{\mathbf{h}}^{*}$ and $\Delta_{0}$ via either $D+1$ equations (in the FP regime) or $D+2$ equations (in the chaotic regime). When these equations are satisfied, the network generalizes the dynamic balance of excitation-inhibition. This balance is dynamic in the sense that without fine-tuning, the macroscopic firing rates in the balance subspace self-adjust to cancel the strong external drive. Depending on the single-neuron transfer function and the strength of the random component of connectivity, balance can take the form of either a stable fixed point, or the more familiar balanced state of chaotic dynamics with local fluctuations propagating in the orthogonal complement.

\section{APPENDIX C: THE CASE OF GAUSSIAN STRUCTURED CONNECTIVITY}

Here we study the mean-field theory for the case in which the structured connectivity is Gaussian. In particular, the elements of $\mathbf{U}$ are taken to be i.i.d. by $\mathcal{N}(0,1)$. The Gaussianity construction greatly simplifies the mean-field expression for $\hat{r}_{k}$ [Eq. (A2)]. In the general case, calculating $\hat{r}_{k}$ requires first averaging $r_{i}=\phi\left(\mathbf{u}_{i}^{T} \hat{\mathbf{h}}+h_{i}^{\perp}\right)$ over the variability in the orthogonal subspace by a Gaussian integral for each neuron $i$ (where $\mathbf{u}_{i}$ is the $i$ th row of $\mathbf{U}$ as above) and then computing a weighted average over the structure of the $k$ th column of $\mathbf{U}$. 


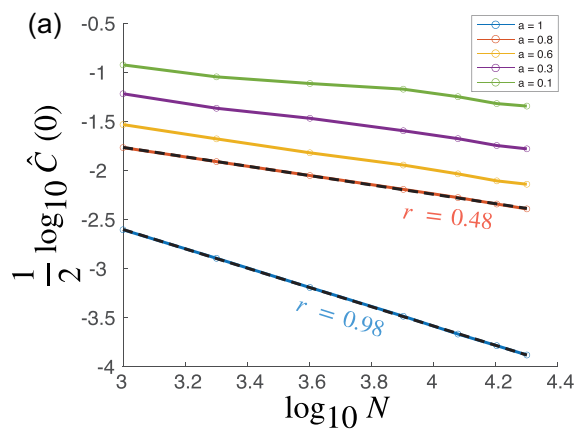

(b) 0.6

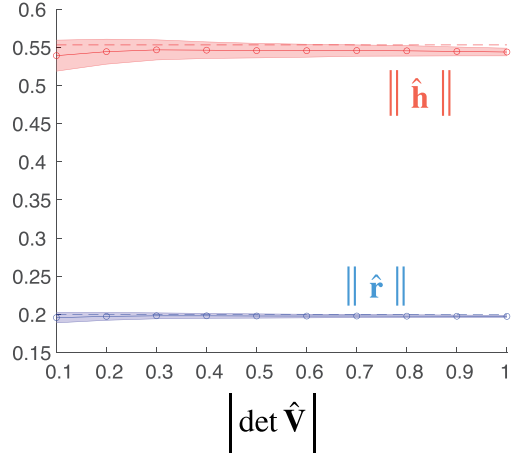

(c)

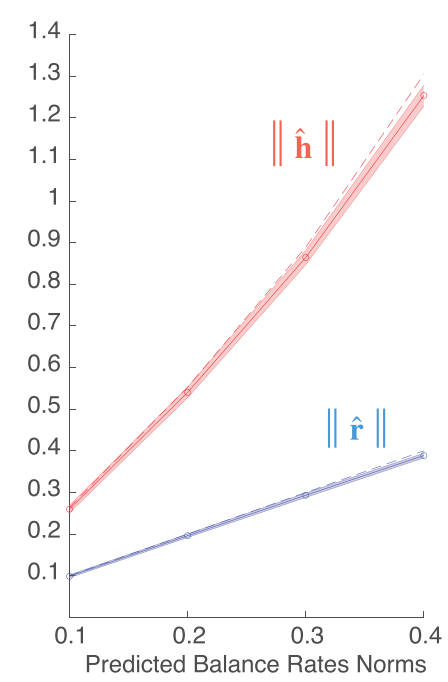

(d)

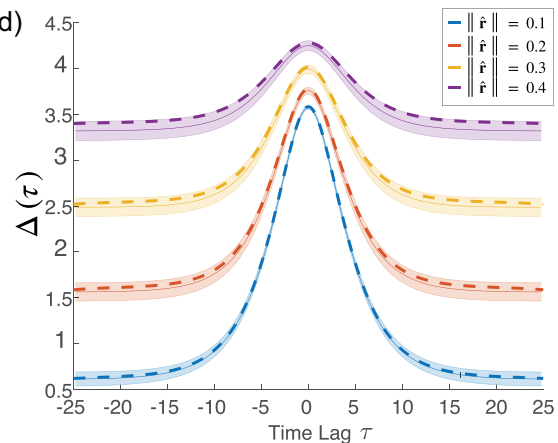

(e)

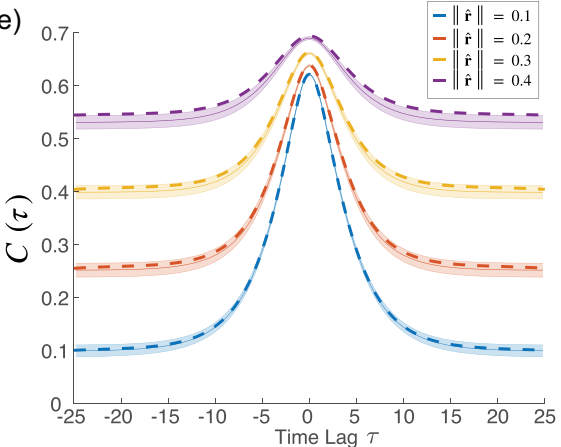

FIG. 5. Fluctuations due to misalignment and dynamic mean-field theory. (a) Simulations of the uniform misalignment parameterization (Appendix F 1) show that misalignment of the structured connectivity increases fluctuations in the balance subspace by an order of magnitude of $O(\sqrt{N})$. The logarithm of the standard deviation of $\hat{\mathbf{r}}(t)$ is plotted against the logarithm of network size. In the case of full alignment $(a=1)$, the slope is almost exactly -1 , indicating that the variance $\hat{C}(0) \sim \frac{1}{N^{2}}$. On the other hand, even mild misalignment $(a=0.8)$ yields a slope of approximately -0.5 , which is consistent with our theory predicting $\hat{C}(0) \sim \frac{1}{N}$ for misaligned structural connectivity. Data points are averages over 10 realizations; black dotted lines are linear fits. [(b)-(d)] In the case of Gaussian structured connectivity, the dynamic mean-field theory for the fluctuations in the orthogonal subspace depend only on the norm of the firing rates in the balance subspace. (b) As described in Appendix F2, we vary the determinant of the alignment matrix $\hat{\mathbf{V}}$ while keeping the predicted norm of the balance firing rates fixed. This figure confirms that indeed in our simulations both the firing rates and the dynamical variables in the balance subspace $(\hat{\mathbf{r}}$ in blue and $\hat{\mathbf{h}}$ in red, respectively) remain constant and very near the dynamic mean-field theory prediction throughout the parameter range. These simulations correspond to Fig. 3(f). Shaded regions display the standard deviation over 20 random realizations of $\hat{\mathbf{V}}$ with fixed singular values, $s_{k}$. (c) We vary the predicted norm of the balance firing rate while fixing the singular values of $\hat{\mathbf{V}}$. Both $\hat{\mathbf{r}}$ in blue and $\hat{\mathbf{h}}$ in red are well predicted by DMFT. (d) The autocorrelation of the microscopic degrees of freedom in the orthogonal complement, $\Delta(\tau) \equiv\left[\left\langle h_{i}^{\perp}(t) h_{i}^{\perp}(t+\tau)\right\rangle\right]$, displayed for the same four values of $\|\hat{\mathbf{r}}\|$ as in panel (c), together with the corresponding DMFT prediction. (e) Same as panel (d) but for the autocorrelation of the single-neuron firing rates, $C(\tau) \equiv\left[\left\langle r_{i}(t) r_{i}(t+\tau)\right\rangle\right] . N=10000$ except where otherwise mentioned.

That is,

$$
\hat{r}_{k}=\frac{1}{N} \sum_{i=1}^{N} u_{i k}\left\langle\phi\left(\sum_{l=1}^{D} u_{i l} \hat{h}_{l}+\sqrt{\Delta_{0}} z\right)\right\rangle_{D z},
$$

In the Gaussian case, the other modes $l \neq k$ contribute Gaussian variability which can simply be added to the variability from the orthogonal subspace, and then $u_{i k}$ can be averaged over as an additional Gaussian. Therefore, $\hat{r}_{k}$ reduces to a double Gaussian integral: one integral for the weighted average over the structure of the $k$ th mode, which is coupled to $\hat{h}_{k}$, and a second Gaussian that combines the remaining $D-1$ structured modes together with the orthogonal complement:

$$
\hat{r}_{k}=\left\langle u \phi\left(u \hat{h}_{k}+\sqrt{\Delta_{0}+\sum_{l \neq k} \hat{h}_{l}^{2} z}\right)\right\rangle_{D u D z} .
$$

The Gaussian integral over $u$ can be performed via integration by parts. Explicitly, one writes $d w \equiv \mathrm{e}^{-\frac{u^{2}}{2}} u d u$ and $v \equiv \phi$, and then $\int d w v=-\int d v w=\int D u \frac{\partial \phi}{\partial u}$. Now the two Gaussians, $u$ and $z$, combine to a single Gaussian integral:

$$
\hat{r}_{k}=\hat{h}_{k}\left\langle\phi^{\prime}\left(\sqrt{\Delta_{0}+\|\hat{\mathbf{h}}\|^{2}} x\right)\right\rangle_{D x}
$$

We find that due to the Gaussianity of $\mathbf{U}, \hat{\mathbf{h}}$ is proportional to $\hat{\mathbf{r}}$. Therefore, the $D$ equations for $\hat{h}_{k}$ reduce to one implicit scalar equation for $\|\hat{\mathbf{h}}\|$ :

$$
\|\hat{\mathbf{r}}\|=\|\hat{\mathbf{h}}\|\left\langle\phi^{\prime}\left(\sqrt{\Delta_{0}+\|\hat{\mathbf{h}}\|^{2}} x\right)\right\rangle_{D x},
$$

where, of course, $\hat{\mathbf{r}}$ is determined to leading order by the balance equations.

The Gaussianity of $\mathbf{U}$ also simplifies the mean-field calculation of the variance in the orthogonal complement, $\Delta_{0}$. In the FP equation [Eq. (B4)], the sum over neurons can be replaced by a Gaussian integral over the balance subspace and combined with the Gaussian integral over the orthogonal 
complement to yield

$$
\Delta_{0}=g^{2}\left\langle\phi^{2}\left(\sqrt{\Delta_{0}+\|\hat{\mathbf{h}}\|^{2}} x\right)\right\rangle_{D x} .
$$

Similarly, we can simplify the dynamic mean-field expression for the total autocorrelation, $C_{T}(\tau) \equiv\left\langle\phi\left(h_{i}(t)\right) \phi\left(h_{i}(t+\tau)\right)\right\rangle$, from Eq. (B11) to

$$
C_{T}(\tau)=\left\langle\left\langle\left.\phi\left(\sqrt{\Delta_{0}-\Delta(\tau)} x+\sqrt{\Delta(\tau)+\|\hat{\mathbf{h}}\|^{2}} y\right)\right|_{D x} ^{2}\right\rangle_{D y} .\right.
$$

Thus, we find that in the Gaussian setting, the DMFT calculation of the autocorrelation in the orthogonal subspace, $\Delta(\tau)$, depends only on the norm in the balance subspace, $\|\hat{\mathbf{h}}\|$. We exploit this in order to calculate $\hat{\mathbf{C}}(\tau)$ [Eq. (14)] as shown in Figs. 4(b)-4(d), and we verify the calculation of $C_{T}(\tau)$ and $\Delta(\tau)$ directly in Fig. 5.

\section{APPENDIX D: FLUCTUATION BALANCE UNDER INCOMPLETE RECURRENT ALIGNMENT}

We return to the balance subspace dynamics in the general setting of incomplete misalignment [Eq. (6)]:

$$
\frac{d \hat{\mathbf{h}}}{d t}=-\hat{\mathbf{h}}+\sqrt{N}\left(\boldsymbol{\Sigma} \hat{\mathbf{V}}^{T} \hat{\mathbf{r}}+\hat{\mathbf{f}}\right)+\frac{1}{\sqrt{N}} \boldsymbol{\Sigma} \mathbf{V}_{\perp}^{T} \mathbf{r}_{\perp} .
$$

Recall that this expression for the dynamics ignores the projection of the random connectivity onto the balance subspace, which is of order of magnitude $O\left(\frac{1}{\sqrt{N}}\right)$.

The dynamics of the fluctuations, $\delta \hat{\mathbf{h}} \equiv \hat{\mathbf{h}}-\langle\hat{\mathbf{h}}\rangle$, are given by

$$
\frac{d \delta \hat{\mathbf{h}}}{d t}=-\delta \hat{\mathbf{h}}+\boldsymbol{\Sigma}\left(\sqrt{N} \hat{\mathbf{V}}^{T} \delta \hat{\mathbf{r}}+\hat{\boldsymbol{\eta}}\right),
$$

where we have written $\hat{\boldsymbol{\eta}} \equiv \frac{1}{\sqrt{N}} \mathbf{V}_{\perp}^{T} \delta \mathbf{r}_{\perp}$.

If the $O(1)$ fluctuations driven by $\hat{\boldsymbol{\eta}}$ are not canceled by corresponding fluctuations in $\delta \mathbf{r}$, then the dynamics of $\delta \hat{\mathbf{h}}$ will have $O(1)$ fluctuations. Such significant fluctuations in the balance subspace would drive $\hat{\mathbf{r}}(t)$ to violate the balance equations, and would in turn generate $O(\sqrt{N})$ fluctuations in $\hat{\mathbf{h}}$. Therefore, balance must suppress these fluctuations, and the $O(1)$ effective feed-forward fluctuations $\hat{\eta}$ will be canceled by fluctuations in the balance subspace activity, $\delta \hat{\mathbf{r}}$. As shown, for example, in Fig. 3(c) in the main text, our simulations confirm that $O(1)$ input fluctuations from the orthogonal complement are canceled to leading order by recurrent balance subspace input, yielding small net input to the balance subspace.

This argument yields a fluctuation balance equation,

$$
\sqrt{N} \hat{\mathbf{V}}^{T} \delta \hat{\mathbf{r}}+\hat{\boldsymbol{\eta}} \approx 0
$$

requiring

$$
\delta \hat{\mathbf{r}} \approx-\frac{1}{\sqrt{N}}\left(\hat{\mathbf{V}}^{T}\right)^{-1} \hat{\boldsymbol{\eta}}
$$

to leading order.

We employ the SVD of $\hat{\mathbf{V}}$ in order to further simplify, writing $\hat{\mathbf{V}}=\mathbf{L S R}^{T}=\sum_{k=1}^{D} L_{k} s_{k} R_{k}^{T}$, where $L_{k}$ and $R_{k}$ are the $k$ th left and right singular vectors of the alignment matrix, respectively. We write $\hat{\eta}_{k}^{R} \equiv R_{k}^{T} \hat{\boldsymbol{\eta}}$, for the effective feedforward input along the $k$ th right singular vector of the alignment matrix, and $\delta \hat{r}_{k}^{L} \equiv L_{k}^{T} \delta \hat{\mathbf{r}}$, for the corresponding rate fluctuations along the $k$ th left singular vector. Then we can re-express the fluctuation balance requirement as

$$
\delta \hat{r}_{k}^{L} \approx-\frac{1}{\sqrt{N}} \frac{\hat{\eta}_{k}^{R}}{s_{k}}
$$

Therefore, for moderate misalignment, i.e., $s_{k} \sim O(1)$, we expect the fluctuations in the balance subspace, $\delta \hat{\mathbf{r}}$, to be $O\left(\frac{1}{\sqrt{N}}\right)$.

To find $\hat{\mathbf{C}}(\tau)$, we observe that by definition $\mathbf{V}_{\perp}$ is orthogonal to $\mathbf{U}$, and it is independent of $\mathbf{J}$ by assumption. Therefore, the fluctuations in the effective feedforward drive, $\hat{\eta}$, can be approximated as a $D$-dimensional Gaussian with matrix of cross-correlation functions:

$$
\left\langle\hat{\boldsymbol{\eta}}(t) \hat{\boldsymbol{\eta}}^{T}(t+\tau)\right\rangle=\frac{\left[C_{T}(\tau)-C_{T}(\infty)\right]}{N} \mathbf{V}_{\perp}^{T} \mathbf{V}_{\perp},
$$

where $C_{T}(\tau)=\left\langle r_{i}(t) r_{i}(t+\tau)\right\rangle$ is the average total autocorrelation function of the firing activity. For notational purposes, we write $C(\tau) \equiv C_{T}(\tau)-C_{T}(\infty)=\left\langle\delta r_{i}(t) \delta r_{i}(t+\tau)\right\rangle$ in the main text [Eq. (14)], for $\delta r_{i}=r_{i}-\left\langle r_{i}\right\rangle$. Note that $C(\tau)$ captures the average single-neuron temporal variability, while the long-time autocorrelation, $C_{T}(\infty)$, captures the spatial variability over single-neuron average firing rates.

To simplify the expression for the covariance of $\hat{\eta}$ and derive an expression for $\hat{\mathbf{C}}(\tau)$, we recall that $\mathbf{V}_{\perp}=\mathbf{V}-\mathbf{U} \hat{\mathbf{V}}$. We note that the particular structure of the columns of $\mathbf{V}_{\perp}$ is unconstrained other than the requirement of being orthogonal to every column of $\mathbf{U}$, but that the $D$-by- $D$ Gram matrix is fully determined by $\hat{\mathbf{V}}$ :

$$
\frac{1}{N} \mathbf{V}_{\perp}^{T} \mathbf{V}_{\perp}=\mathbf{I}_{D \times D}-\hat{\mathbf{V}}^{T} \hat{\mathbf{V}}=\mathbf{R}\left(\mathbf{I}_{D \times D}-\mathbf{S}^{2}\right) \mathbf{R}^{T} .
$$

Interestingly, this implies that the effective feedforward drive is uncorrelated in the basis given by the right-singular vectors of $\hat{\mathbf{V}}$, i.e.,

$$
\left\langle\hat{\eta}_{k}^{R}(t) \hat{\eta}_{j}^{R}(t+\tau)\right\rangle=\delta_{k, j} C(\tau)\left(1-s_{k}^{2}\right) .
$$

We thus find, by applying Eq. (D5), that the corresponding balance subspace rate fluctuations projected along the left singular vectors, $L_{k}$, are uncorrelated:

$$
\left\langle\delta \hat{r}_{k}^{L}(t) \delta \hat{r}_{j}^{L}(t+\tau)\right\rangle=\delta_{k, j} \frac{C(\tau)}{N} \frac{1-s_{k}^{2}}{s_{k^{2}}} .
$$

In other words, the left singular vectors of the alignment matrix, $L_{k}$, are the eigenvectors of the covariance, $\hat{\mathbf{C}}$, or equivalently, the principal components of the macroscopic fluctuations in the balance subspace. The corresponding eigenvalues, i.e., the variances, are $\frac{C(\tau)}{N} \frac{1-s_{k}^{2}}{s_{k^{2}}}$.

The total variance in the balance subspace, $\operatorname{Tr} \hat{\mathbf{C}}(0)=$ $\left\langle\delta \hat{\mathbf{r}}^{T} \delta \hat{\mathbf{r}}\right\rangle$, is

$$
\operatorname{Tr} \hat{\mathbf{C}}(0)=\frac{C(0)}{N} \sum_{k}\left(\frac{1}{s_{k}^{2}}-1\right) .
$$


We can change bases to return to the standard basis of the balance subspace (the columns of $\mathbf{U}$ ):

$$
\hat{\mathbf{C}}(\tau)=\frac{C(\tau)}{N} \mathbf{L}\left(\mathbf{S}^{-2}-\mathbf{I}_{D \times D}\right) \mathbf{L}^{T},
$$

which is equivalent to the result presented in Eq. (14) in the main text.

\section{APPENDIX E: THE CASE OF COMPLETE NONALIGNMENT}

In this section, we study the case of a complete nonalignment, in which at least one of the singular values of the alignment matrix, $\hat{\mathbf{V}}=\frac{1}{N} \mathbf{U}^{T} \mathbf{V}$, is small $\left[s_{j} \sim O\left(\frac{1}{\sqrt{N}}\right)\right]$.

As discussed in the previous section, the Gram matrix of $\mathbf{V}_{\perp}=\mathbf{V}-\mathbf{U} \hat{\mathbf{V}}$ is determined by by $\hat{\mathbf{V}}$ and given by $\frac{1}{N} \mathbf{V}_{\perp}^{T} \mathbf{V}_{\perp}=\mathbf{R}\left(\mathbf{I}_{D \times D}-\mathbf{S}^{2}\right) \mathbf{R}^{T}$. Therefore, we can find some $N$-by- $D$ matrix $\mathbf{U}_{\perp}$ whose columns have norm $\sqrt{N}$ and are all orthogonal to each column of $\mathbf{U}$, and write

$$
\mathbf{V}_{\perp}=\mathbf{U}_{\perp} \sqrt{\mathbf{I}_{D \times D}-\mathbf{S}^{2}} \mathbf{R}^{T} .
$$

Therefore, we can write $\mathbf{M}=\frac{1}{\sqrt{N}} \mathbf{U} \boldsymbol{\Sigma} \mathbf{V}^{T}$ as

$$
\mathbf{M}=\frac{1}{\sqrt{N}} \mathbf{U} \boldsymbol{\Sigma} \mathbf{R}\left(\mathbf{S} \mathbf{L}^{T} \mathbf{U}^{T}+\sqrt{\mathbf{I}_{D \times D}-\mathbf{S}^{T}} \mathbf{U}_{\perp}\right) .
$$

Thus, we see that in this scenario, with $s_{j} \sim O\left(\frac{1}{\sqrt{N}}\right)$, there is one macroscopic subspace, $\mathbf{u}_{j}^{L} \equiv \mathbf{U} L_{j}$, which does not send any recurrent feedback to the balance subspace, and therefore as we will show, the activity in this subspace is unconstrained by the balance equations.

To see this, we first rescale the balance subspace dynamics [Eq. (D1)] by $\boldsymbol{\Sigma}^{-1}$ and write: $\mathbf{x} \equiv \boldsymbol{\Sigma}^{-1} \hat{\mathbf{h}}$ and $\mathbf{y} \equiv \boldsymbol{\Sigma}^{-1} \hat{\mathbf{f}}$, yielding dynamics

$$
\frac{d \mathbf{x}}{d t}=-\mathbf{x}+\sqrt{N}\left(\hat{\mathbf{V}}^{T} \hat{\mathbf{r}}+\mathbf{y}\right)
$$

to leading order, where we have momentarily ignored the effective feedforward input, which we will return to below.

Next, we rotate the rescaled dynamics to the basis of right singular vectors of $\hat{\mathbf{V}}, x_{k}^{R} \equiv R_{k}^{T} \mathbf{x}$, while projecting the balance subspace activity to the basis of left singular vectors, $\hat{r}_{k}^{L} \equiv$ $L_{k}^{T} \hat{\mathbf{r}}$, yielding $D$ dynamical equations:

$$
\frac{d x_{k}^{R}}{d t} \approx-x_{k}^{R}+\sqrt{N}\left(s_{k} \hat{r}_{k}^{L}+y_{k}^{R}\right) .
$$

Modes with nonzero alignment, $s_{k} \sim O(1)$, yield a linear balance equation:

$$
\hat{r}_{k}^{L}=-\frac{y_{k}^{R}}{s_{k}},
$$

which is a restatement of the $D$-dimensional balance equations in the main text, using the identity $y_{k}^{R}=R_{k}^{T} \boldsymbol{\Sigma}^{-1} \hat{\mathbf{f}}$ [Eq. (7)].

For the unaligned mode, however, balance requires small external drive, $\hat{y}_{j}^{R} \sim O\left(\frac{1}{\sqrt{N}}\right)$. That is, we require that the strong external drive in the balance subspace have no projection on the $j$ th right singular vector of the alignment matrix. We note that this requirement is an extension of our assumption throughout that $\hat{\mathbf{f}}$ is chosen in order to allow a set of balance equations with obtainable firing rates.
Therefore, we can write the balance subspace firing rates as

$$
\hat{\mathbf{r}}=-\sum_{k \neq j} \frac{y_{k}^{R}}{s_{k}} L_{k}+\hat{r}_{j}^{L}(t) L_{j},
$$

where $r_{j}^{L}(t)$ is unconstrained by the balance equations.

We now turn to the fluctuation dynamics of $j$ th mode. We write $s_{j}=\frac{s}{\sqrt{N}}$ and $y_{j}^{R}=\frac{y}{\sqrt{N}}$, and we have

$$
\frac{d x_{j}^{R}}{d t}=-x_{j}^{R}+s \hat{r}_{j}^{L}+y+\hat{\eta}_{j}^{R},
$$

where $\hat{\eta}_{j}^{R}=\frac{1}{\sqrt{N}} R_{j}^{T} \mathbf{V}_{\perp}^{T} \mathbf{r}_{\perp} \sim O(1)$, which in the large- $N$ limit is a Gaussian process with autocorrelation $\left\langle\eta_{j}^{R}(t) \eta_{j}^{R}(t+\tau)\right\rangle=$ $C_{T}(\tau)$ following Eq. (D8). This equation predicts $O(1)$ fluctuations in both $x_{j}^{R}$ and $\hat{r}_{j}^{L}$, but the self-consistent solution involves all the other modes and is beyond the scope of this work.

In the limit of zero alignment, $s=y=0$, the $x_{j}^{R}$ are expected to be simply a Gaussian process with autocorrelation given by

$$
\left\langle x_{j}^{R}(t) x_{j}^{R}(t+\tau)\right\rangle=\frac{\Delta(\tau)}{g^{2}},
$$

where $\Delta(\tau) \equiv\left\langle h_{i}^{\perp}(t) h_{i}^{\perp}(t+\tau)\right\rangle$.

Note, however, that the fluctuations in $\hat{\mathbf{h}}=\mathbf{\Sigma} \mathbf{x}$ will have nontrivial impact on the microscopic fluctuations in the orthogonal subspace, and therefore $\Delta(\tau)$ can no longer be derived from the DMFT theory above [Eq. (B12)], except in the limit of $\sigma_{j} \ll 1$. As shown in Ref. [21], in that limit the fluctuations in $\hat{h}_{j}^{R}$ do not impact $\Delta(\tau)$ to leading order, and therefore this regime exhibits a passive coherent chaos with macroscopic fluctuations that are driven in a purely feedforward-like manner by the microscopic chaos in the orthogonal complement via the nonaligned mode of the structured connectivity.

\section{APPENDIX F: CONSTRUCTING CONNECTIVITY MATRICES WITH INCOMPLETE ALIGNMENT}

\section{Constructing arbitrary low-rank structure with uniform misalignment}

As a concrete example, we study a specific form of misalignment in which the alignment matrix is scaled down uniformly across the balance subspace modes. Concretely, for this section we fix the rank $D$ and then in order to construct the structured connectivity, $\mathbf{M}=\frac{1}{\sqrt{N}} \mathbf{U} \boldsymbol{\Sigma} \mathbf{V}^{T}$, we first fix the diagonal of $\boldsymbol{\Sigma}$ to be non-negative numbers (usually we set them to be all ones for simplicity), and then sample the elements of $\mathbf{U}$ independently from a standard Gaussian distribution. In order to define $\mathbf{V}$, we first construct an arbitrary orthonormal $D$-by- $D$ matrix, $\hat{\mathbf{A}}$, which will be the alignment matrix in the case of full alignment. To construct $\hat{\mathbf{A}}$, we generate random pairs of orthonormal vectors of dimension $D$ which each serve as the real and imaginary parts of an eigenvector of $\hat{\mathbf{A}}$. We associate to each pair a uniform random phase constrained to have negative real part. For odd $D$, we add a real eigenvector with eigenvalue -1 . Explicitly, we generate orthonormal vec- 
tors $\mathbf{q}_{r e}^{k}$ and $\mathbf{q}_{i m}^{k}$ for $k=1 \ldots\lfloor$ L $/ 2\rfloor$ and $\theta_{k} \sim U\left[\frac{\pi}{2}, \frac{3 \pi}{2}\right]$, and then define

$$
\begin{gathered}
\mathbf{z}^{k}=\mathbf{q}_{r e}^{k}+i \mathbf{q}_{i m}^{k}, \\
\mathbf{z}^{D-k}=\mathbf{q}_{r e}^{k}-i \mathbf{q}_{i m}^{k} .
\end{gathered}
$$

For odd $D$, we set $\mathbf{z}^{(D+1) / 2}$ to be a random real orthonormal vector and $\theta_{(D+1) / 2}=-\pi$. Next we define $\mathbf{Z}$ to be the matrix of column vectors consisting of $\mathbf{z}^{k}$ and $\boldsymbol{\Theta}$ to be the diagonal matrix with $\theta_{k}$ along the diagonal, and finally we have

$$
\hat{\mathbf{A}}=\mathbf{Z} \mathrm{e}^{\Theta} \mathbf{Z}^{T},
$$

where the ${ }^{T}$ here means conjugate transpose. Thus, $\hat{\mathbf{A}}$ is a random orthonormal $D$-by- $D$ matrix with eigenvalues constrained to the left half of the complex plane. This matrix will define the structure of true recurrence within the balance subspace, which will be additionally scaled by a scalar parameter to adjust the extent of alignment as follows.

We construct an $N$-by- $D$ matrix $\mathbf{A}_{\perp}$, with $\mathbf{A}_{\perp}^{T} \mathbf{A}_{\perp}=N \mathbf{I}_{D \times D}$ such that $\mathbf{U}^{T} \mathbf{A}_{\perp}=\mathbf{0}$. Finally, we define $\mathbf{V}$ as

$$
\mathbf{V} \equiv a \mathbf{U} \hat{\mathbf{A}}+\sqrt{1-a^{2}} \mathbf{A}_{\perp} .
$$

Thus, the parameter $a$ scales down the alignment matrix uniformly:

$$
\hat{\mathbf{V}}=\frac{1}{N} \mathbf{U}^{T} \mathbf{V}=a \hat{\mathbf{A}} .
$$

With this parametrization of the alignment matrix, we have $\hat{\mathbf{r}}^{*}=-\frac{1}{a} \boldsymbol{\Sigma}^{-1} \hat{\mathbf{f}}$ for the solution to the balance equations. Plugging this into the mean-field equation for $\left\|\hat{\mathbf{h}}^{*}\right\|$ in the case of Gaussian structure [Eq. (C4)], we have

$$
\left\|\hat{\mathbf{h}}^{*}\right\| \int_{-\infty}^{\infty} D z \phi^{\prime}\left(\sqrt{\Delta_{0}+\left\|\hat{\mathbf{h}}^{*}\right\|^{2}} z\right)=\frac{r_{0}}{a}\left\|\boldsymbol{\Sigma}^{-1} \hat{\mathbf{f}}\right\| .
$$

This equation is coupled with the mean-field equation for $\Delta_{0}$, Eq. (C5), and we solve this pair of equations and compare to simulations in Fig. 5.

\section{Constructing heterogeneous misalignment}

Recall that our dynamic mean-field theory prediction for the total temporal variance in the balance subspace is

$$
\left\langle\delta \hat{\mathbf{r}}^{T} \delta \hat{\mathbf{r}}\right\rangle=\operatorname{Tr} \hat{\mathbf{C}}(0)=\frac{C(0)}{N} \sum_{k}\left(\frac{1}{s_{k}^{2}}-1\right)
$$

as derived in Sec. IV of the main text. Note that in the parametrization of Appendix F1, all $D$ singular values of $\hat{\mathbf{V}}$ are given by $a$. In order to verify our theory in a more generic framework, we fix the left and right singular vectors of $\hat{\mathbf{V}}$ and then construct the $D$ singular values. In order to nevertheless restrict simulations to a single parameter, we adjust the absolute value of the determinant of $\hat{\mathbf{V}}$, and then set the sequence of $D$ singular values to decay exponentially from 1 while constraining their product.

Explicitly, given a fixed $|\operatorname{det} \hat{\mathbf{V}}|=\prod_{k=0}^{D-1} s_{k}$, we write

$$
l_{s} \equiv \frac{1}{D} \ln |\operatorname{det} \hat{\mathbf{V}}|=\frac{1}{D} \sum_{k=1}^{D} \ln s_{k} .
$$

We then define $s_{k}=\exp \left(2 k \frac{l_{s}}{(D-1)}\right)$ for $k=0, \ldots, D-1$. This indeed gives $\prod_{k=0}^{D-1} s_{k}=\exp \left(\frac{2 \ln |\operatorname{det} \hat{\mathbf{V}}|}{D(D-1)} \sum_{0}^{D-1} k\right)=|\operatorname{det} \hat{\mathbf{V}}|$.

For this parametrization, we can write the total temporal variance as

$$
\left\langle\delta \hat{\mathbf{r}}^{T} \delta \hat{\mathbf{r}}\right\rangle=\frac{C(0)}{N}\left(\frac{1-|\operatorname{det} \hat{\mathbf{V}}|^{-\frac{4}{D-1}}}{1-|\operatorname{det} \hat{\mathbf{V}}|^{-\frac{4}{D(D-1)}}}-D\right) .
$$

This is the theory curve displayed alongside simulation results in Figs. 4(b)-4(e) and Figs. 5(b)-5(e).

In practice, we choose sets of singular vectors of $\hat{\mathbf{V}}$ by sampling from random Gaussians and orthogonalizing. If any of the resulting eigenvalues of $\hat{\mathbf{V}}$ have positive real part we resample. This works effectively for up to at least $D=5$ but fails for larger $D$.

\section{APPENDIX G: BIOLOGICAL RELEVANT EXAMPLES WITH MORE DETAILS}

\section{Degenerate excitation-inhibition balance example for unequal population size}

As in the main text, we consider an $E I$ network in which the synaptic weight depends only on the presynaptic neuron: $J_{E I}=J_{I I}=J_{I}$ and $J_{E E}=J_{I E}=J_{E}$, such that the structured connectivity becomes rank 1: $\frac{\sigma}{\sqrt{N}} \mathbf{u}_{0} \mathbf{v}_{0}^{T}$. We now consider a network with $N_{I}=\gamma N$ inhibitory neurons, and the remaining $N_{E}=(1-\gamma) N$ are excitatory. The structured connectivity is

$$
\begin{gathered}
\mathbf{u}_{0}=\left(\begin{array}{c}
\mathbf{1}_{N_{E}} \\
\mathbf{1}_{N_{I}}
\end{array}\right), \\
\mathbf{v}_{0}=\frac{1}{\sigma}\left(\begin{array}{c}
J_{E} \mathbf{1}_{N_{E}} \\
-J_{I} \mathbf{1}_{N_{I}}
\end{array}\right), \\
\sigma=\sqrt{(1-\gamma) J_{E}^{2}+\gamma J_{I}^{2}},
\end{gathered}
$$

where $\mathbf{1}_{N_{x}}$ is the uniform column vector of length $N_{X}$.

The alignment between $\mathbf{u}_{0}$ and $\mathbf{v}_{0}$ is given by

$$
\hat{v}=\frac{(1-\gamma) J_{E}-\gamma J_{I}}{\sigma} .
$$

Inhibition dominance and network stability will require that $J_{I} \geqslant \frac{1-\gamma}{\gamma} J_{E}$. At the critical boundary, $\hat{v}=0$.

External alignment requires that the external drive be uniform, $\mathbf{f}=r_{0} \mathbf{1}$, and the balance equation yields population average firing

$$
\hat{r}=\frac{r_{0}}{\gamma J_{I}-(1-\gamma) J_{E}} .
$$

The external drive can be made to compensate for diminished alignment by scaling $r_{0} \propto \hat{v} \sigma=\gamma J_{I}-(1-\gamma) J_{E}$. In this case, even as inhibition is weakened and alignment decreases, the balanced fixed point remains unchanged to leading order. In this situation, $\hat{C}(t)=\langle\hat{r}(t) \hat{r}(t+\tau)\rangle$ is given by

$$
\hat{C}(\tau)=\frac{C(\tau)}{N} \frac{\gamma(1-\gamma)\left(J_{E}+J_{I}\right)^{2}}{\left[(1-\gamma) J_{E}-\gamma J_{I}\right]^{2}} .
$$

We see that the size of macroscopic fluctuations increases as the net strength of inhibitory synapses, $N_{I} J_{I}=\gamma N J_{I}$, 
approaches the net strength of excitatory synapses, $N_{E} J_{E}=$ $(1-\gamma) N J_{E}$.

\section{Heterogeneous in and out degrees}

Here we consider the case of heterogeneity in both out and in degrees, with possible correlations between them. We have a single inhibitory population in which each neuron $i$ is randomly connected via $K_{i}^{\text {in }}$ incoming connections, and has $K_{i}^{\text {out }}$ randomly chosen outgoing connections, both with average $K$, and where each nonzero synapse has weight $-\frac{J}{\sqrt{N}}$. Such a connectivity structure can be approximated by a deterministic rank-1 structure given by

$$
M_{i j}=-\frac{J}{\sqrt{N}} \frac{K_{i}^{\text {in }} K_{j}^{\text {out }}}{K N} .
$$

We define the relative in and out degrees as $k_{i}^{\alpha} \equiv \frac{K_{i}^{\alpha}}{K}$, and the mean square of the relative in and out degrees is $\left\langle k_{\alpha}^{2}\right\rangle$, for $\alpha \in$ \{in, out $\}$. Then, we can write

$$
\begin{gathered}
\mathbf{u}_{0}=\frac{\mathbf{k}^{\text {in }}}{\sqrt{\left\langle k_{\text {in }}^{2}\right\rangle}}, \\
\mathbf{v}_{0}=-\frac{\mathbf{k}^{\text {out }}}{\sqrt{\left\langle k_{\text {out }}^{2}\right\rangle}}, \\
\sigma_{0}=\sqrt{\left\langle k_{\text {in }}^{2}\right\rangle\left\langle k_{\text {out }}^{2}\right\rangle} J p,
\end{gathered}
$$

where $p \equiv \frac{K}{N}$.

The scalar alignment in this case is

$$
\hat{v}=-\frac{1}{N} \frac{\mathbf{k}^{\text {in }^{T}} \mathbf{k}^{\text {out }}}{\sqrt{\left\langle k_{\text {in }}^{2}\right\rangle\left\langle k_{\text {out }}^{2}\right\rangle}}=-\frac{1+c}{\sqrt{\left\langle k_{\text {in }}^{2}\right\rangle\left\langle k_{\text {out }}^{2}\right\rangle}},
$$

where $c=\left\langle\left(k_{i}^{\text {in }}-1\right)\left(k_{i}^{\text {out }}-1\right)\right\rangle$ is the covariance of the relative in and out degrees. We find that if the in and out degrees are uncorrelated, then

$$
\hat{v}=-\frac{1}{\sqrt{\left\langle k_{\text {in }}^{2}\right\rangle\left\langle k_{\text {out }}^{2}\right\rangle}}
$$

and the extent of alignment decreases with increasing breadth of the degree distributions. Correlations between in and out degrees increase the alignment and in the extreme case of fully correlated degrees, the absolute alignment remains large and depends only on the relative breadth of the two distributions, for example, $\hat{v}=-1$ for fully correlated degree distributions with identical variances.

External alignment requires that $\mathbf{f}=r_{0} \mathbf{u}=r_{0} \frac{\mathbf{k}^{\text {in }}}{\sqrt{\left\langle k_{\text {in }}^{2}\right\rangle}}$, similar to Ref. [37]. The balance equation gives

$$
\hat{r}=-\frac{r_{0}}{\hat{v} \sigma}=\frac{r_{0}}{(1+c) J p} .
$$

We find that if in and out degrees are anticorrelated the balance rates will be driven up.

Note that in this setting the balance subspace is defined by the in degrees: $\hat{r}=\frac{1}{N}{\frac{\mathbf{k}^{\text {in }}}{\sqrt{\left\langle k_{\text {in }}^{2}\right\rangle}}}^{T} \mathbf{r}$. The population average firing rate, $\bar{r} \equiv \frac{1}{N} \mathbf{1}^{T} \mathbf{r}$, will be approximately equal to $\bar{r} \approx \frac{1}{\sqrt{\left\langle k_{\text {in }}^{2}\right\rangle}} \hat{r}$. The population average external drive is scaled down by the same factor, $\bar{r}_{0} \equiv \frac{1}{N} \mathbf{1}^{T} \mathbf{f}=\frac{r_{0}}{\sqrt{\left\langle k_{\text {in }}^{2}\right\rangle}}$, so that the balance fixed point is not impacted by the degree distributions themselves and only affected by the in to out correlations.

The macroscopic fluctuations, however, will increase with broader degree distributions even in the absence of correlations:

$$
\hat{C}(\tau)=\frac{C(\tau)}{N}\left(\left\langle k_{\text {in }}^{2}\right\rangle\left\langle k_{\text {out }}^{2}\right\rangle-1\right) .
$$

The resulting fluctuations in the population average will be $\bar{C}(\tau)=\frac{C(\tau)}{N}\left(\left\langle k_{\text {out }}^{2}\right\rangle-\frac{1}{\left\langle k_{\text {in }}^{2}\right\rangle}\right)$, such that they increase with the breadth of each degree distribution.

For correlated in and out degrees, we find

$$
\hat{C}(\tau)=\frac{C(\tau)}{N}\left(\frac{\left\langle k_{\text {in }}^{2}\right\rangle\left\langle k_{\text {out }}^{2}\right\rangle}{(1+c)^{2}}-1\right),
$$

so that positive correlations between in and out degrees decrease shared fluctuations while negative correlations amplify them.
[1] C. van Vreeswijk and H. Sompolinsky, Chaos in neuronal networks with balanced excitatory and inhibitory activity, Science 274, 1724 (1996).

[2] C. van Vreeswijk and H. Sompolinsky, Chaotic balanced state in a model of cortical circuits. Neural Comput. 10, 1321 (1998).

[3] N. Brunel, Dynamics of sparsely connected networks of excitatory and inhibitory spiking neurons, J. Comput. Neurosci. 8, $183(2000)$

[4] A. Renart, J. D. Rocha, P. Bartho, L. Hollender, N. Parga, A. Reyes, and K. D. Harris, The asynchronous state in cortical circuits, Science 327, 587 (2010).

[5] W. R. Softky and C. Koch, The highly irregular firing of cortical cells is inconsistent with temporal integration of random EPSPs. J. Neurosci. 13, 334 (1993).
[6] A. S. Ecker, P. Berens, G. A. Keliris, M. Bethge, N. K. Logothetis, and A. S. Tolias, Decorrelated neuronal firing in cortical microcircuits, Science 327, 584 (2010).

[7] A. S. Ecker, P. Berens, R. J. Cotton, M. Subramaniyan, G. H. Denfield, C. R. Cadwell, S. M. Smirnakis, M. Bethge, and A. S. Tolias, State dependence of noise correlations in macaque primary visual cortex, Neuron 82, 235 (2014).

[8] M. R. Cohen and A. Kohn, Measuring and interpreting neuronal correlations, Nat. Neurosci. 14, 811 (2011).

[9] B. Doiron, A. Litwin-Kumar, R. Rosenbaum, G. K. Ocker, and K. Josić, The mechanics of state-dependent neural correlations, Nat. Neurosci., 19, 383 (2016). 
[10] A. Roxin, N. Brunel, D. Hansel, G. Mongillo, and C. van Vreeswijk, On the distribution of firing rates in networks of cortical neurons. J. Neurosci. 31, 16217 (2011).

[11] J. Kadmon, J. Timcheck, and S. Ganguli, Predictive coding in balanced neural networks with noise, chaos and delays, Adv. Neural Inf. Proc. Sys. 33, 16677 (2020).

[12] D. Hansel and C. van Vreeswijk, The mechanism of orientation selectivity in primary visual cortex without a functional map. J. Neurosci. 32, 4049 (2012).

[13] C. Pehlevan and H. Sompolinsky, Selectivity and sparseness in randomly connected balanced networks. PLoS One 9, e89992 (2014).

[14] C. van Vreeswijk and H. Sompolinsky, Irregular activity in large networks of neurons, in Methods and Models in Neurophysics: Lecture Notes of the Les Houches Summer School 2003 (Elsevier, Amsterdam, 2005).

[15] Y. Roudi and P. E. Latham, A balanced memory network. PLoS Comput. Biol. 3, e141 (2007).

[16] G. Mongillo, S. Rumpel, and Y. Loewenstein, Inhibitory connectivity defines the realm of excitatory plasticity, Nat. Neurosci. 21, 1463 (2018).

[17] H. R. Wilson and J. D. Cowan, Excitatory and inhibitory interactions in localized populations of model neurons. Biophys. J. 12, 1 (1972).

[18] O. Harish and D. Hansel, Asynchronous rate chaos in spiking neuronal circuits. PLoS Comput. Biol. 11, e1004266 (2015).

[19] J. Kadmon and H. Sompolinsky, Transition to Chaos in Random Neuronal Networks, Phys. Rev. X 5, 041030 (2015).

[20] H. Sompolinsky, A. Crisanti, and H. J. Sommers, Chaos in Random Neural Networks, Phys. Rev. Lett. 61, 259 (1988).

[21] I. D. Landau and H. Sompolinsky, Coherent chaos in a recurrent neural network with structured connectivity, PLoS Comput. Biol. 14, e1006309 (2018).

[22] R. Darshan, C. Van Vreeswijk, and D. Hansel, Strength of Correlations in Strongly Recurrent Neuronal Networks, Phys. Rev. X 8, 031072 (2018).

[23] T. Hayakawa and T. Fukai, Spontaneous and stimulus-induced coherent states of critically balanced neuronal networks, Phys. Rev. Res. 2, 013253 (2020).

[24] A. Rivkind and O. Barak, Local Dynamics in Trained Recurrent Neural Networks, Phys. Rev. Lett. 118, 258101 (2017).

[25] F. Mastrogiuseppe and S. Ostojic, Linking connectivity, dynamics, and computations in recurrent neural networks, Neuron 99, 609 (2018)

[26] M. Helias, T. Tetzlaff, and M. Diesmann, The correlation structure of local neuronal networks intrinsically results from recurrent dynamics, PLoS Comput. Biol. 10, e1003428 (2014).

[27] F. Schuessler, A. Dubreuil, F. Mastrogiuseppe, S. Ostojic, and O. Barak, Dynamics of random recurrent networks with correlated low-rank structure, Phys. Rev. Research 2, 13111 (2020).

[28] A. Roxin, The role of degree distribution in shaping the dynamics in networks of sparsely connected spiking neurons. Front. Comput. Neurosci. 5, 8 (2011).

[29] C. Baker, V. Zhu, and R. Rosenbaum, Nonlinear stimulus representations in neural circuits with approximate excitatoryinhibitory balance, PLoS Comput. Biol. 16, e1008192 (2020).

[30] I. D. Landau, The impact of structural connectivity on dynamic balance in cortical circuits, Ph.D. thesis, Hebrew University of Jerusalem, 2020.

[31] J. Aljadeff, M. Stern, and T. Sharpee, Transition to Chaos in Random Networks with Cell-Type-Specific Connectivity, Phys. Rev. Lett. 114, 088101 (2015).

[32] S. Ostojic, Two types of asynchronous activity in networks of excitatory and inhibitory spiking neurons. Nat. Neurosci. 17, 594 (2014)

[33] R. Rosenbaum and B. Doiron, Balanced Networks of Spiking Neurons with Spatially Dependent Recurrent Connections, Phys. Rev. X 4, 021039 (2014).

[34] C. Huang, D. A. Ruff, R. Pyle, R. Rosenbaum, M. R. Cohen, and B. Doiron, Circuit models of low-dimensional shared variability in cortical networks, Neuron 101, 337 (2019).

[35] C. Ebsch and R. Rosenbaum, Spatially extended balanced networks without translationally invariant connectivity, J. Math. Neurosci. 10, 1 (2020).

[36] R. Pyle and R. Rosenbaum, Highly connected neurons spike less frequently in balanced networks, Phys. Rev. E 93, 040302 (2016).

[37] I. D. Landau, R. Egger, V. J. Dercksen, M. Oberlaender, and H. Sompolinsky, The impact of structural heterogeneity on excitation-inhibition balance in cortical networks, Neuron 92, 1 (2016).

[38] R. Darshan, W. E. Wood, S. Peters, A. Leblois, and D. Hansel, A canonical neural mechanism for behavioral variability, Nat. Commun. 8, 15415 (2017).

[39] R. Rosenbaum, M. A. Smith, A. Kohn, J. E. Rubin, and B. Doiron, The spatial structure of correlated neuronal variability, Nat. Neurosci. 20, 107 (2017).

[40] D. Sussillo and L. F. Abbott, Generating coherent patterns of activity from chaotic neural networks. Neuron 63, 544 (2009).

[41] B. Barzel and A. L. Barabási, Universality in network dynamics, Nat. Phys. 9, 673 (2013).

[42] C. Hens, U. Harush, S. Haber, R. Cohen, and B. Barzel, Spatiotemporal signal propagation in complex networks, Nat. Phys. 15, 403 (2019).

[43] Code repository, https://github.com/ilandau/Incomplete_ Alignment_and_Macroscopic_Fluctuations. 